\title{
Energy Budget of a Small Convectively Driven Marginal Sea: The Gulf of Eilat/Aqaba (Northern Red Sea)*
}

\author{
ELI BITON \\ Department of Physics, Israel Oceanographic and Limnological Research, Haifa, Israel \\ HEZI GILDOR \\ Institute of Earth Sciences, Hebrew University, Jerusalem, Israel
}

(Manuscript received 9 October 2013, in final form 23 April 2014)

\begin{abstract}
Oceanic model simulations and model-based energy budget calculations have been used to quantitatively study the relative importance of different processes affecting the Gulf of Eilat/Aqaba dynamics and hydrographic conditions. Large seasonality among various energetic pathways is observed. Similar to other oceanic waters, a significant amount of energy is stored in the potential energy (PE) that is predominately in the form of background potential energy (BPE). During September-March PE/BPE gains energy by raising the center of mass due to atmospheric cooling. During April-August, PE decreases its strength due to the exchange of light, warmer water from the northern Red Sea through the Strait of Tiran. The available potential energy (APE) is stored in baroclinic modes that are linked to the along-gulf temperature gradient. During AprilAugust, the APE is sustained by baroclinic energy flux through the straits, resulting from advective density fronts into the gulf, and during September-March, the APE is sustained by the differential heating at the surface. Part of the APE is converted into kinetic energy (KE) by vertical buoyancy flux and in smaller quantity to background potential energy by isopycnal mixing. The conversion from APE is the only source of $\mathrm{KE}$, where losses are related to the work done by pressure at the straits and energy dissipation. The wind stress work subtracts energy from the buoyancy-driven flow, which explained by the opposite surface flow and mean wind directions. The authors found that temperature and salinity variations contribute to the dynamics, via the APE-KE conversion, by a ratio of 10:1.
\end{abstract}

\section{Introduction}

The Gulf of Eilat/Aqaba (hereinafter the gulf) is a small, convectively driven, marginal sea connected to the northern Red Sea through the Straits of Tiran (hereinafter straits) (Fig. 1). The general circulation of the gulf consists of relatively warm and fresh Red Sea surface water $\left(23.2^{\circ} \mathrm{C} ; 40.4 \mathrm{psu}\right)$ that enters from the south through the straits as a surface layer and flows northward, whereas the relatively cold and saline water of the gulf exits as the bottom layer of the exchange flow

\footnotetext{
* Supplemental information related to this paper is available at the Journals Online website: http://dx.doi.org/10.1175/JPO-D-130220.s1.

Corresponding author address: Eli Biton, National Institute of Oceanography, Tel-Shikmona, P.O. Box 8030, Haifa 31080, Israel. E-mail: elib@ocean.org.il
}

at the straits $\left(21.6^{\circ} \mathrm{C} ; 40.7 \mathrm{psu}\right)$. The annual-mean net evaporation rate (evaporation rate minus precipitation rate) and net heat loss flux at the surface are $\sim 1.6 \mathrm{~m} \mathrm{yr}^{-1}$ and $\sim 55 \mathrm{~W} \mathrm{~m}^{-2}$ (Ben-Sasson et al. 2009; Biton and Gildor 2011b), respectively. Year-round, the arid mountains on both side of the gulf steer the prevailing northerly wind ( $~ 80 \%$ of the time) along the main axis of the gulf (Berman et al. 2003; Afargan and Gildor 2014, manuscript submitted to J. Mar. Syst.) opposite to the mean flow at the surface. The surface flow is composed of a chain of eddies along the main axis of the gulf as suggested by numerical models (Berman et al. 2000; Biton and Gildor 2011b) and current measurements along the basin (Manasrah et al. 2004). The gulf's hydrography exhibits strong seasonal variability (Fig. 2): the surface cooling that taking place from September to March leads to gravitational instability and hence to thermally driven vertical mixing (hereinafter termed the mixing phase), which is followed by a restratification due to 


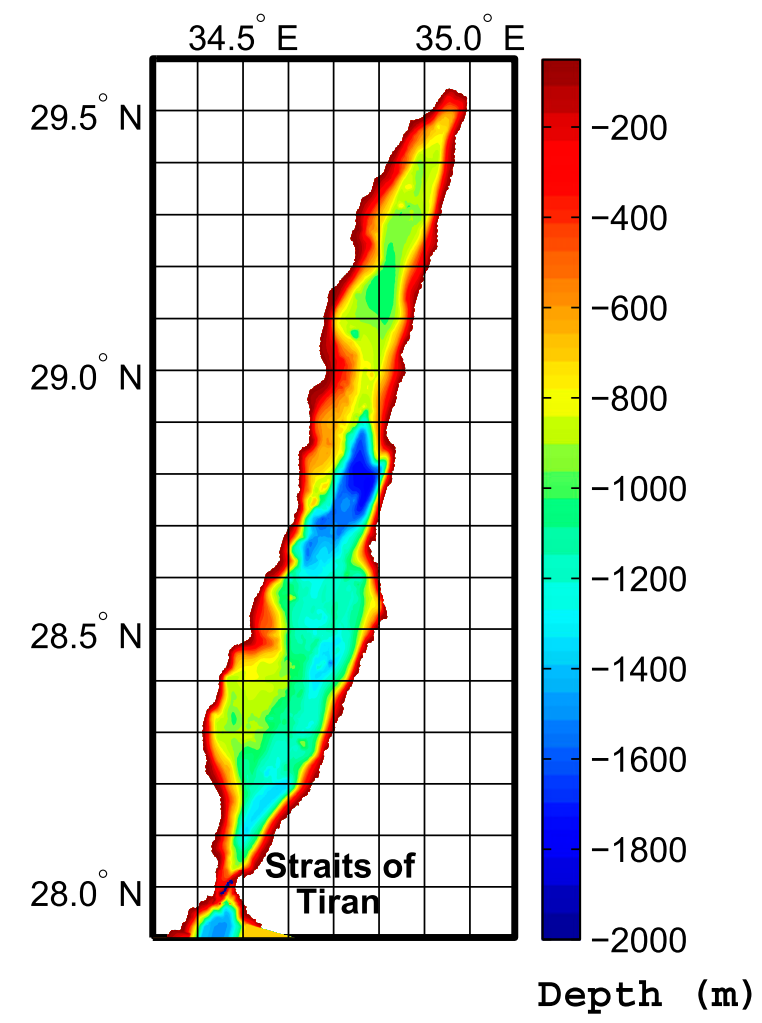

FIG. 1. Domain of the OGCM and bathymetry of the gulf based on Hall and Ben-Avraham (1973). Also indicated are the Straits of Tiran.

advected warm water from the northern Red Sea between April and August (hereinafter termed the restratification phase) (Biton and Gildor 2011b).

Recently, Biton and Gildor (2011b,c) have used an oceanic model to study the gulf's seasonal stratification evolution in response to the flow through the straits, surface fluxes (evaporation rate and heat flux), and wind stress. They showed that the relatively large amount of heat advected through the straits during the restratification phase is responsible for most of the observed warming and deepening of the surface layer of the gulf (Fig. 2). Specifically, the model results suggest that during the several months following the winter mixing, the upper $\sim 150 \mathrm{~m}$ of the entire gulf is replaced by the influx of northern Red Sea surface water. During the mixing phase a small amount of advected heat from the straits and large heat loss to the atmosphere lead to efficient erosion of the stratification at the surface (Fig. 2). With the aid of a simple two-layer hydraulic model for the exchange flow through the straits, Biton and Gildor (2011a) proposed a mechanistic explanation for the time-dependent seasonal variations described above that takes into account the hydraulic conditions in the straits and their coupling with the stratification conditions in the gulf and in the northern Red Sea. The proposed mechanism successfully accounted for the heat budget of the gulf and revealed the role played by wintertime deep/intermediate water formation in the general circulation of the gulf. A more detailed description of the conditions in the gulf can be found in Biton and Gildor (2011b) and references therein.

Our goals in the present paper are as follows: 1) to quantitatively investigate the seasonal responses of the gulf's dynamics and hydrography to (i) the relative contributions of the exchange properties at the straits, (ii) the thermohaline fluxes and wind forces at the surface, and (iii) internal processes in the gulf (friction, diapycnal mixing, convection, and vertical adiabatic motions); and 2) to quantitatively differentiate between the relative contributions of temperature and salinity seasonal variations to the dynamics and hydrography. To achieve these goals, we track pathways associated with the gulf's energy budget, using the oceanic general circulation model described in Biton and Gildor (2011b). In E. Biton and H. Gildor (2014, unpublished manuscript), a companion paper to our work here, we suggest a mechanism that provides a link between the above forcings and the horizontal circulation pattern that includes the formation of eddies along the gulf.

The manuscript is organized as follows: In section 2 , we describe shortly the model, where the model equations and model setup that are relevant to the calculation of the energy budget are discussed in more detail in appendix A. In section 3, we formulate an energetic framework for our model equations that is based on previous studies, which can be easily generalized to other marginal seas or open systems (e.g., Mediterranean Sea, Red Sea, and Labrador Sea). In section 4, we present and discuss the energy budget results for the gulf.

\section{Model description and equations}

We use the Massachusetts Institute of Technology general circulation model (MITgcm; Marshall et al. 1997b,a) as described in Biton and Gildor (2011b) (but with a different equation of state). The model domain includes the whole gulf and ends $\sim 20 \mathrm{~km}$ south of the straits. The horizontal resolution is $300 \mathrm{~m}$, with 32 unevenly distributed vertical levels, most of which are concentrated in the upper $300 \mathrm{~m}$ to capture the complex dynamics in the upper ocean. The time step is $100 \mathrm{~s}$. At the southern end of the domain, there is a sponge layer in which temperature and salinity are relaxed to monthlymean profiles. At the surface, monthly-mean wind stress, net evaporation, and heat flux are imposed. Wind stress is based on Berman et al. (2000) and has no spatial variability (Fig. 3c), and the surface thermohaline fluxes are 

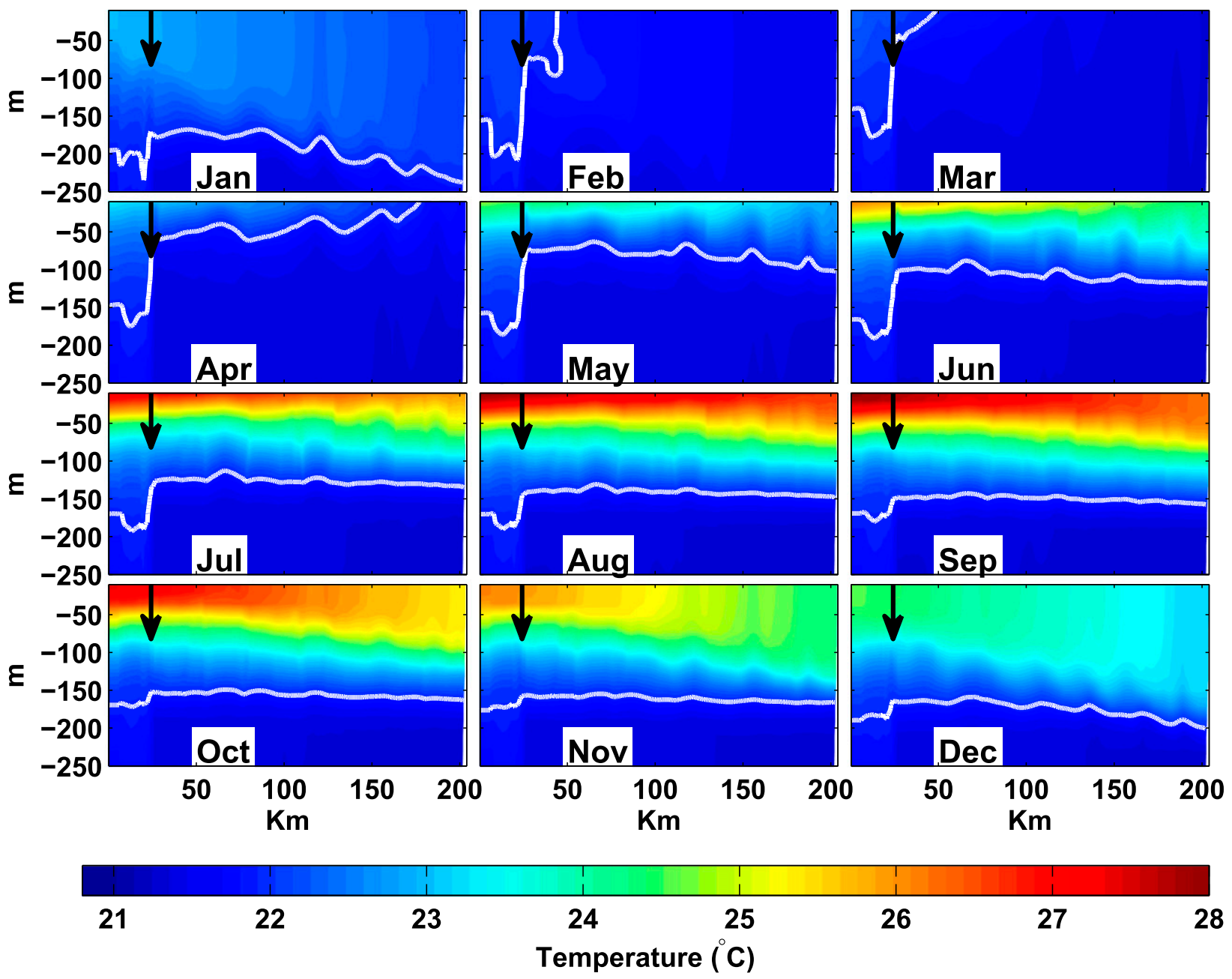

FIG. 2. Modeled, monthly, zonally averaged, potential temperature down to $250 \mathrm{~m}$ along the central south-north axis of the gulf and the northern Red Sea $\left({ }^{\circ} \mathrm{C}\right)$. Black arrows indicate the location of the Enterprise Passage in the straits, and white lines indicate the $21.9^{\circ} \mathrm{C}$ isotherm, which separates the gulf intermediate water from the surface water (Biton and Gildor 2011b). Large excessive heat loss form the surface of the gulf from September to March slowly erodes the stratification, where the warming conditions from April to August at the surface layer are largely driven by the exchange flow through the straits during this time (section 1).

derived using the iterative procedure described in Biton and Gildor (2011b) (Figs. 3a,b). The model solves an approximated form of the full set of the thermodynamic and momentum equations, known as the hydrostatic primitive equations (HPEs). We use incompressible fluid (density is a conserved value, i.e., $D \rho / D t=0$ ), Boussinesq approximation (for which all terms multiplied by the density are replaced by the reference value $\rho_{o}$, with the exception of the gravitational force), and linear freesurface $\eta$. Unlike the model described in Biton and Gildor (2011b), here we use a linear equation of state for seawater density that allows the calculation of the energy budget more easily. However, this change has a negligible effect on the model results shown in Biton and Gildor (2011b). The model reached a repeating seasonal cycle and simulated the conditions in the gulf reasonably well compared to existing observations (Biton and Gildor 2011b). A more detailed description of the model can be found in Biton and Gildor (2011b).

\section{Energy budget calculations for marginal seas}

The energy within the marginal sea is stored as kinetic energy $\left(\mathrm{KE}^{V}\right)$, available potential energy $\left(\mathrm{APE}^{V}\right)$, background potential energy $\left(\mathrm{BPE}^{V}\right)$ and internal energy $\left(\mathrm{IE}^{V}\right)$ (e.g., Winters et al. 1995), where the superscript $V$ denotes volume-integrated values. These energy reservoirs are influenced by 1) internal exchanges between the different energy reservoirs and 2) the influx/ outflux of energy at the basin's boundaries including exchange at the sea surface, through the straits, and at the solid boundaries. In sections $3 \mathrm{a}-\mathrm{c}$, we will develop 
(a)

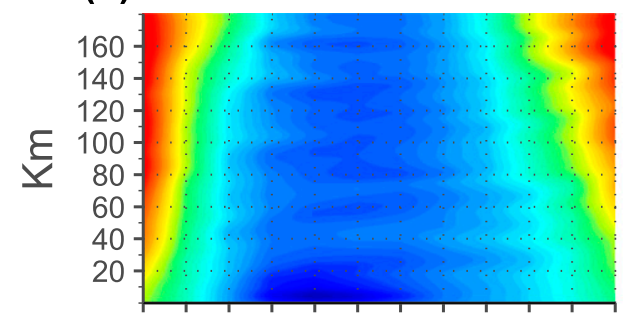

(b)

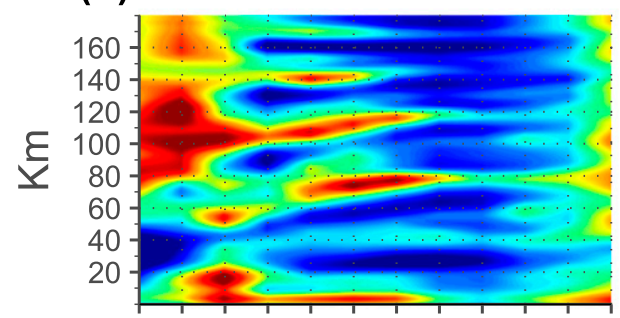

(c)

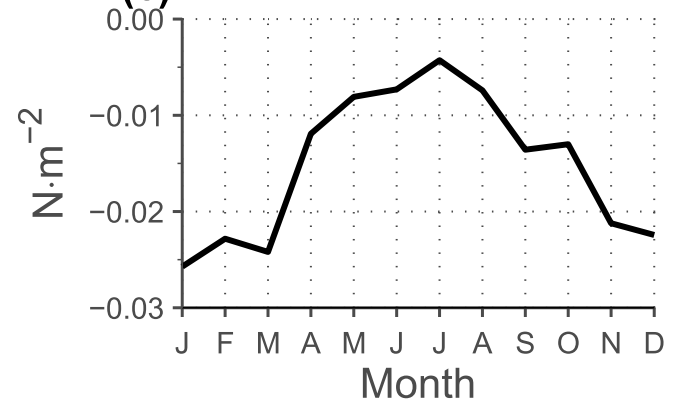

(d)
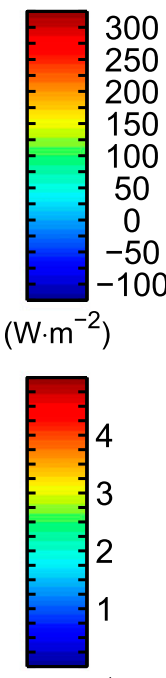

$\left(\mathrm{m} \cdot\right.$ year $\left.^{-1}\right)$

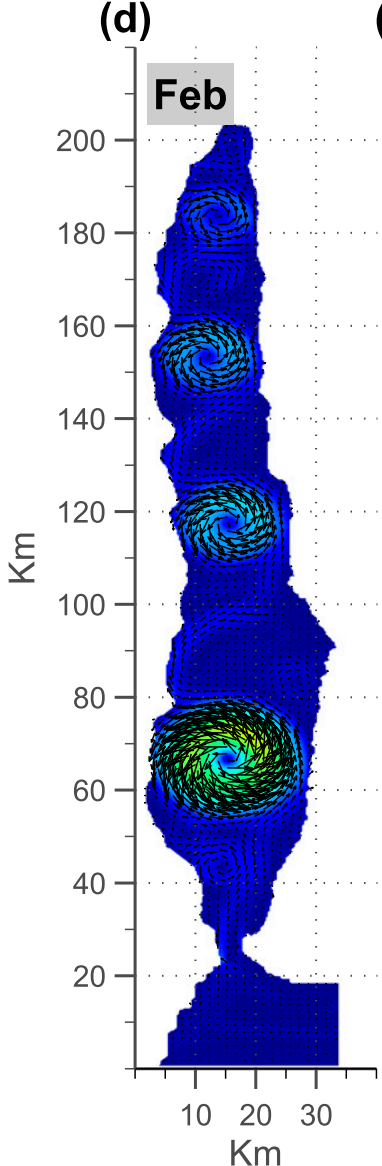

(e)

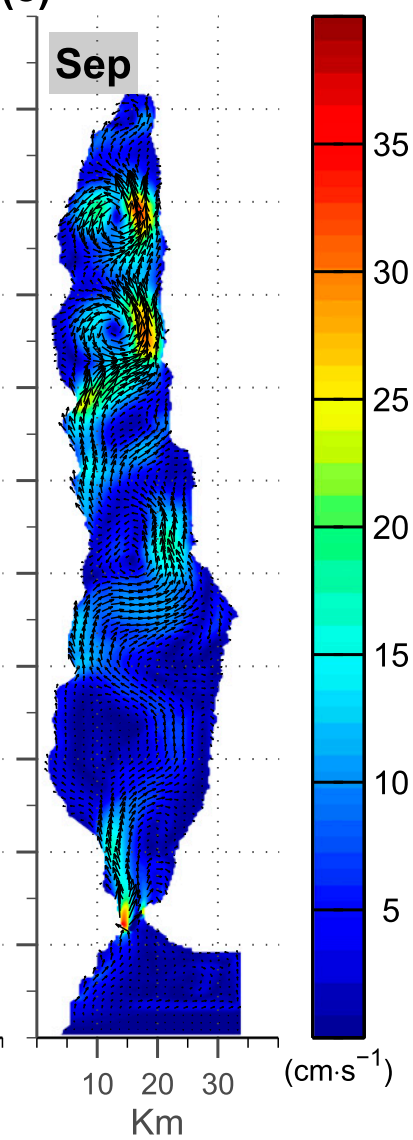

35

30

25

20

FIG. 3. Monthly, zonally averaged values ( $x$ axis) along the main axis of the gulf ( $y$ axis, increasing northward) for the (a) net heat flux $\left(\mathrm{W} \mathrm{m}^{-2}\right)$ and $(\mathrm{b})$ net evaporation $\left(\mathrm{m} \mathrm{yr}^{-1}\right)$. These air-sea fluxes were calculated by our model results, based on the iterative procedure described in Biton and Gildor (2011b). (c) Monthly, average meridional wind stress $\left(\mathrm{N} \mathrm{m}^{-2}\right)$, where negative values indicate northerlies winds. The modeled monthly-mean surface currents $\left(\mathrm{cm} \mathrm{s}^{-1}\right)$ for (d) September and (e) February. Chain of eddies are seen along the main axis of the basin as in previous studies (section 1).

the equations, which are specifically suited to our modeling setup (section 2) following previous studies that developed a similar set of equations (e.g., Winters et al. 1995; Huang 1998; Kuhlbrodt et al. 2007; Hughes et al. 2009), while including the lateral energy exchanges at the straits and incorporating a realistic bathymetry. In addition, we calculate separately the contributions of salinity and temperature to the potential energy (PE) budget, enabling us to trace the energy pathways more accurately based on model calculations. In this paper, however, as we use the incompressible model we do not calculate the internal energy reservoir; therefore, $\mathrm{IE}^{V}$ only appears when transfers to and from mechanical energy are required. More detailed description of $\mathrm{IE}^{V}$ calculations can be found in Kuhlbrodt et al. (2007) and Tailleux (2009). As we note in section 2, and as described in more detail in appendix A, we use an approximated form of the momentum equations, which might not be accurate for energy budget calculations when using the convective adjustment scheme (Hughes et al. 2009). Therefore, Hughes et al. (2009) suggested that nonhydrostatic model simulations should be used for accurate energy calculations. Yet, here we are using the nonlocal $K$-profile parameterization scheme for vertical mixing of Large et al. (1994) (appendix A), which unifies the treatments of a variety of unresolved processes involved in vertical mixing. Unlike the convective adjustment scheme, the $K$-profile parameterization scheme does not vertically mix the water immediately to eliminate statistically unstable density gradients. Therefore, we expect that the calculated energy pathways will be better resolved when using the $K$-profile scheme. In fact, comparison of our model results and the associated energetic calculations as shown in section 4 to similar calculations based on a nonhydrostatic simulation showed minor differences (see the supplementary information). 
We therefore proceed with an approximated form of equations as described in section 2 and in appendix A.

\section{a. Evolution of the kinetic energy reservoir}

In the model the vertical momentum equation is not fully resolved, and the vertical velocity $w$ is calculated diagnostically based on the horizontal currents $\mathbf{V}_{h}$ and the continuity relation (appendix A) because we are using the ocean primitive equations. Therefore, the kinetic energy density (per unit volume) of the flow is derived from the horizontal currents alone as follows,

$$
\mathrm{KE}=\frac{\rho_{0}}{2}\left|\mathbf{V}_{h}\right|^{2},
$$

and the kinetic energy reservoirs, stored in the entire basin, is given by

$$
\mathrm{KE}^{V}=\int_{V} \mathrm{KE} d V
$$

where $V$ is the volume of the marginal sea measured from the strait's cross section. A time derivative of (2) gives the evolution of the kinetic energy reservoir:

$$
\frac{\partial \mathrm{KE}^{V}}{\partial t}=\frac{\partial}{\partial t} \int_{V} \mathrm{KE} d V=\int_{V} \frac{\partial \mathrm{KE}}{\partial t} d V
$$

where the last equality is possible as we are using a linear free surface, where the equations are vertically integrated up to the surface reference level (i.e., $z=H$ ), short of reaching the actual surface height $\eta$, and therefore the basin's volume $V$ is constant in time. The time derivative of the kinetic energy density of the flow that appears in the above integral is given by

$$
\frac{\partial \mathrm{KE}}{\partial t}=\rho_{0} \mathbf{V}_{h} \cdot \frac{\partial \mathbf{V}_{h}}{\partial t} .
$$

Substituting the time derivatives of the horizontal currents from the horizontal momentum (A1) will yield

$$
\begin{aligned}
\frac{\partial \mathrm{KE}}{\partial t}= & -\rho_{0} \mathbf{V}_{h} \cdot\left(\mathbf{V} \cdot \nabla \mathbf{V}_{h}\right)-\mathbf{V}_{h} \cdot \nabla_{h} P^{\prime} \\
& +\rho_{0} \mathbf{V}_{h} \cdot\left(\boldsymbol{\nabla} \mathbf{A} \nabla \mathbf{V}_{h}\right)-w \frac{\partial P^{\prime}}{\partial z}+w \frac{\partial P^{\prime}}{\partial z},
\end{aligned}
$$

where the term $w\left(\partial P^{\prime} / \partial z\right)$ is added and removed on the right-hand side of (5). Using continuity (A3) and hydrostatic relation (A2), we get an expression for the evolution of the kinetic energy density:

$$
\frac{\partial \mathrm{KE}}{\partial t}=-\nabla\left[\mathbf{V}\left(\mathrm{KE}+P^{\prime}\right)-\mathbf{A} \cdot \nabla \mathrm{KE}\right]-g \rho^{\prime} w-\rho_{0} \epsilon,
$$

where $\rho_{0} \epsilon$ is the kinetic energy dissipation rate per unit volume, and $\epsilon$ is given by

$$
\epsilon=A_{h}\left(\left|\frac{\partial \mathbf{V}_{h}}{\partial x}\right|^{2}+\left|\frac{\partial \mathbf{V}_{h}}{\partial y}\right|^{2}\right)+A_{z}\left|\frac{\partial \mathbf{V}_{h}}{\partial z}\right|^{2} .
$$

Integrating (6) over the basin volume, as in (2), will give the evolution of the stored kinetic energy in the marginal sea:

$$
\begin{aligned}
\frac{\partial \mathrm{KE}^{V}}{\partial t}= & -\int_{V} g \rho^{\prime} w d V-\int_{V} \rho_{0} \epsilon d V \\
& -\int_{\Omega}\left(\mathbf{V K E}+\mathbf{V} P^{\prime}-\mathbf{A} \cdot \nabla \mathrm{KE}\right) \cdot \mathbf{n}^{\Omega} d \Omega,
\end{aligned}
$$

where $\boldsymbol{\Omega}$ is the basin's envelope surface, and $\mathbf{n}^{\mathbf{\Omega}}$ is a unit vector normal to that surface. Contributors to the kinetic energy reservoir are expressed by the energy exchanges with other energy reservoirs [volume integrals in (8)] and external energy inputs at the basin's envelope (last integral). The first volume integral in (8) is the reversible buoyancy flux of the flow, and the second term expresses the dissipation of kinetic energy (Winters et al. 1995). From left to right, the terms in the surface integral express the fluxes of advective kinetic energy, pressure work, and the work done by boundary stress terms (here only including wind stress at the sea surface and the bottom drag). Using the relation of pressure anomaly (A6), and the boundary conditions (A7) and (A11)-(A12) we finally get

$$
\begin{aligned}
\frac{\partial \mathrm{KE}^{V}}{\partial t}= & \int_{\Omega^{H}}\left(-w \mathrm{KE}-\rho_{0} g \eta w+\mathbf{V}_{h} \cdot \tau\right)^{H} d \Omega^{H} \\
& -\oint_{\Omega_{S}}\left(\mathbf{V}_{h} \mathrm{KE}+\mathbf{V}_{h} P^{\prime}-A_{h} \nabla_{h} \mathrm{KE}\right)^{S} \cdot \mathbf{n}^{S} d \Omega^{S} \\
& -\int_{\Omega_{B}} \rho_{0} C_{d}\left|\mathbf{V}_{h}^{B}\right|^{3} d \Omega^{B}-\int_{V} g \rho^{\prime} w d V-\int_{V} \rho_{0} \epsilon d V,
\end{aligned}
$$

where the superscripts $H, S$, and $B$ indicate quantities at the surface, at the strait cross section, and at the bottom, respectively. We keep the circled surface integral at the strait in case more than one passage connect between the marginal sea and the open ocean (as is the case of the Straits of Tiran), or the domain has other lateral open boundaries. Following previous works (e.g., Hughes et al. 2009), we give shortened notations for all quantities in the above equation, which simplifies to

$$
\begin{aligned}
\frac{\partial \mathrm{KE}^{V}}{\partial t}= & \phi_{k a}^{H}+\phi_{k w}^{H}+\phi_{k \tau}^{H}+\phi_{k a}^{S}+\phi_{k w}^{S} \\
& +\phi_{k \tau}^{S}+\phi_{k \tau}^{B}+\phi_{p k}^{V}+\epsilon^{V},
\end{aligned}
$$


where the $k$ subscript indicates contributors to the kinetic energy budget, and the $a, w$, and $\tau$ subscripts indicate the quantities of advection, pressure work, and boundary stress, respectively. The last two terms in (10) account for the volume integrals of the reversible buoyancy flux of the flow $\phi_{p k}^{V}$ and the dissipation of kinetic energy $\epsilon^{V}$.

\section{b. Evolution of the potential energy}

The gravitational potential energy reservoirs, stored in the entire basin, are given by

$$
\mathrm{PE}^{V}=\int_{V} \rho g z d V
$$

where $\rho=\rho^{\prime}+\rho_{0}$. Taking the time derivative of (11), and substituting the density anomaly (A4), yields the evolution of the potential energy reservoir:

$$
\frac{\partial \mathrm{PE}^{V}}{\partial t}=\int_{V} g z \frac{\partial \rho^{\prime}}{\partial t} d V=\int_{V} g z\left(\mathbf{\nabla} \mathbf{K} \nabla \rho^{\prime}-\mathbf{V} \cdot \nabla \rho^{\prime}\right) d V .
$$

Substituting $\quad g z \nabla \mathbf{K} \nabla \rho^{\prime}=g \nabla \mathbf{K} z \nabla \rho^{\prime}-g K_{z}\left(\partial \rho^{\prime} / \partial z\right)=$ $\nabla g z\left[K_{h} \nabla_{h} \rho^{\prime}+K_{z}\left(\partial \rho^{\prime} / \partial z\right) \mathbf{z}\right]-g K_{z}\left(\partial \rho^{\prime} / \partial z\right)$ and $-g z \mathbf{V} \cdot \nabla \rho^{\prime}=$ $-\nabla\left(\rho^{\prime} g z \mathbf{V}\right)+g \rho^{\prime} w$ [where we used continuity (A3) to derive the second relation], (12) can be written as follows:

$$
\begin{aligned}
\frac{\partial \mathrm{PE}^{V}}{\partial t}= & \int_{V} g \rho^{\prime} w d V-\int_{V} g K_{z} \frac{\partial \rho^{\prime}}{\partial z} d V \\
& +\oint_{\Omega}\left[g z\left(K_{h} \nabla_{\mathbf{h}} \rho^{\prime}+K_{z} \frac{\partial \rho^{\prime}}{\partial z} \mathbf{z}\right)-\rho^{\prime} g z \mathbf{V}\right] \cdot \mathbf{n}^{\Omega} d \Omega .
\end{aligned}
$$

Similar to the kinetic energy, contributors to the potential energy reservoir break into internal energy exchanges [volume integrals in (13)] and external energy inputs at the basin's envelope (last integral). The first volume integral in (13) is the reversible buoyancy flux of the flow that appears in the opposite sign in (9); therefore, this term defines the energy transfer between potential and kinetic energy reservoirs. The second volume integral in (13) is the conversion rate from internal to potential energy due to vertical diffusion or the irreversible diapycnal mixing term (Winters et al. 1995; Hughes et al. 2009). Yet, as we mentioned in section 3, the internal energy is not calculated in the model and is not exchanged energy with the potential energy or any other form of energy in the model. The terms in the surface integral in (13) are the diffusive and advective fluxes of potential energy across the domain's envelope surface. Using the boundary conditions (A7), (A13), and (A16), we get

$$
\begin{aligned}
\frac{\partial \mathrm{PE}^{V}}{\partial t}= & \int_{\Omega^{H}}\left(H B_{0} \rho_{0}-\rho^{\prime} g H w\right)^{H} d \Omega^{H} \\
& +\oint_{\Omega^{S}}\left(g z K_{h} \nabla_{h} \rho^{\prime}-\rho^{\prime} g z \mathbf{V}\right)^{S} \cdot \mathbf{n}^{S} d \Omega^{S} \\
& +\int_{V} g \rho^{\prime} w d V-\int_{V} g K_{z} \frac{\partial \rho^{\prime}}{\partial z} d V,
\end{aligned}
$$

where $B_{o}$ is the buoyancy flux at the surface that is given by (A17).

Similar to (9), (14) is written in shorthand as

$\frac{\partial \mathrm{PE}^{V}}{\partial t}=\phi_{p B_{0}}^{H}+\phi_{p a}^{H}+\phi_{p d}^{S}+\phi_{p a}^{S}-\phi_{p k}^{V}+\phi_{p d}^{V}$,

where the $p$ subscript indicates contributors to the potential energy budget, and the $B_{o}, a$, and $d$ subscripts indicate the contributions of surface buoyancy flux, advection, and diffusion, respectively. As it indicated in appendix B, part of the terms that appear in the potential energy equations (14) and (15) are sensitive to the choice of the vertical reference level (at which $z=0$ ). Previous studies had placed the vertical reference level either at the bottom of the ocean (e.g., Winters et al. 1995; Huang 1998; Hughes et al. 2009), as done here, or at the surface of the ocean (e.g., Cessi et al. 2014), in which the formulations look somewhat different. However, as we explain in appendix B, the vertical reference level can be arbitrarily chosen without any real effect on the dynamics or on the interpretation in what follows.

As we mentioned in section 1 , we are also interested in the relative contributions of the temperature and salinity time tendencies to the potential energy budget for two reasons: 1) Biton and Gildor (2011b) concluded that the dynamics of the gulf are driven mainly by temperature variations, while salinity variations have a negligible contribution. The energetic approach can quantitatively support this conclusion, and 2) while temperature and salinity are prognostic variables, density is obtained diagnostically in most general circulation models [using the equation of state, e.g., (A5)]. Separating the contributions of salinity and temperature to the energy budget will allow the energy pathways to be traced more accurately based on the model calculations. Substituting (A5) into (11) will yield

$$
\begin{aligned}
\mathrm{PE}^{V}= & \int_{V} g z \rho_{0} d V-\int_{V} g z \rho_{0} \alpha\left(T-T_{0}\right) d V \\
& +\int_{V} g z \rho_{0} \beta\left(S-S_{0}\right) d V \\
= & \mathrm{PER}^{V}+\mathrm{PET}^{V}+\mathrm{PES}^{V},
\end{aligned}
$$

where $\mathrm{PER}^{V}$ is the potential energy stored in a homogeneous basin with the reference water density $\rho_{0}$, and $\mathrm{PET}^{V}$ 
and $\operatorname{PES}^{V}$ are the potential energy contributions due to density anomalies caused by the variations in temperature and salinity, respectively. Equations for the evolution of
$\mathrm{PET}^{V}$ and $\mathrm{PES}^{V}$ can be derived using continuity (A3), time-dependent derivatives of temperature and salinity (A4), and the boundary conditions (A13)-(A15) as follows:

$$
\begin{aligned}
\frac{\partial \mathrm{PET}^{V}}{\partial t}= & \int_{\Omega^{H}} g \rho_{0} \alpha\left[\frac{H Q^{*}}{C_{p} \rho_{0}}+H w\left(T-T_{0}\right)\right]^{H} d \Omega^{H}+\oint_{\Omega^{S}}\left[-g \rho_{0} \alpha z K_{h} \nabla_{h} T+g \rho_{o} \alpha z\left(T-T_{o}\right) \mathbf{V}\right]^{S} \cdot \mathbf{n}^{S} d \Omega^{S} \\
& -\int_{V} g \rho_{0} \alpha\left(T-T_{0}\right) w d V+\int_{V} g \rho_{0} \alpha K_{z} \frac{\partial T}{\partial z} d V, \text { and } \\
\frac{\partial \mathrm{PES}^{V}}{\partial t}= & \int_{\Omega^{H}} g \rho_{0} \beta\left[H \cdot S \cdot E^{*}-H w\left(S-S_{0}\right)\right]^{H} d \Omega^{H}+\oint_{\Omega^{S}}\left[g \rho_{0} \beta z K_{h} \nabla_{h} S-g \rho_{0} \beta z\left(S-S_{0}\right) \mathbf{V}\right]^{S} \cdot \mathbf{n}^{S} d \Omega^{S} \\
& +\int_{V} g \rho_{0} \beta\left(S-S_{0}\right) w d V-\int_{V} g \rho_{0} \beta K_{z} \frac{\partial S}{\partial z} d V,
\end{aligned}
$$

which are written in abbreviated form as

$$
\begin{aligned}
\frac{\partial \mathrm{PET}^{V}}{\partial t}= & \phi T_{p B_{0}}^{H}+\phi T_{p a}^{H}+\phi T_{p d}^{S}+\phi T_{p a}^{S} \\
& -\phi T_{p k}^{V}+\phi T_{p d}^{V}, \text { and } \\
\frac{\partial \mathrm{PES}^{V}}{\partial t}= & \phi S_{p B_{0}}^{H}+\phi S_{p a}^{H}+\phi S_{p d}^{S}+\phi S_{p a}^{S}-\phi S_{p k}^{V}+\phi S_{p d}^{V}
\end{aligned}
$$

Note that the sum of corresponding terms in (19) and (20) is equal to their equivalent term in (15) (e.g., $\phi_{p B_{0}}^{H}=\phi T_{p B_{0}}^{H}+\phi S_{p B_{0}}^{H}$ ).

\section{c. Evolution of the background and available potential energies}

Background potential energy is the minimum of potential energy possible for the basin, achieved when the density field in the entire domain is adiabatically restored into its natural buoyant level (known also as the reference state). Therefore, in the reference state all the isopycnals in the basin are level and the stratification is stable everywhere. Following Winters et al. (1995), the background potential energy reservoir is expressed by

$$
\mathrm{BPE}^{V}=\int_{V} \rho g z^{*} d V
$$

where $z^{*}$ is defined as the geopotential height of a mass element in the reference state. As we are using realistic bathymetry, $z^{*}$ is given implicitly by the following relation:

$$
\int_{V} G\left[\rho\left(\mathbf{x}^{\prime}, t\right)-\rho(\mathbf{x}, t)\right] d V^{\prime}=\int_{b(x, y)}^{z^{*}(\mathbf{x}, t)} A(z) d z .
$$

The term $A(z)$ in (22) is the area of the basin at a given depth, and $G(\zeta)$ is a Heaviside step function (Winters et al. 1995) as follows:

$$
G(\zeta)= \begin{cases}0, & \zeta<0 \\ \frac{1}{2}, & \zeta=0 \\ 1, & \zeta>0\end{cases}
$$

The integral on the right-hand side of (22) is the volume of the basin measured from the bottom to the reference level $z^{*}$, which by the definition of $z^{*}$ is equal to the volume of the parcels of water that are denser or have an equal density with respect to a given density $\rho$ [the integral on the left-hand side of (22)]. Similar to previous studies (e.g., Hughes et al. 2009), in case of a flat bottom ( $A$ is constant and $b=0$ ), relation (22) becomes

$$
z^{*}=\frac{1}{A} \int_{V} G\left[\rho\left(\boldsymbol{x}^{\prime}, t\right)-\rho(\boldsymbol{x}, t)\right] d V^{\prime} .
$$

Differentiating (21), noting that the integration volume is constant as before, the evolution of the background potential energy reservoir is

$$
\frac{\partial \mathrm{BPE}^{V}}{\partial t}=\int_{V} g\left(\frac{\partial \rho}{\partial t} z^{*}+\rho \frac{\partial z^{*}}{\partial t}\right) d V
$$

By taking the time derivative of (22), it can be shown that $\partial z^{*} / \partial t=0$ as follows: Noting that the volume of the gulf is constant, the time derivative of the lefthand side of (22) that is given by $\int_{V}\left\{\partial G\left[\rho\left(\mathbf{x}^{\prime}, t\right)-\rho(\mathbf{x}, t)\right] /\right.$ $\partial t\} d V^{\prime}=\int_{V}\left\{\partial G / \partial\left[\rho\left(\mathbf{x}^{\prime}, t\right)-\rho(\mathbf{x}, t)\right]\right\}\left\{\partial\left[\rho\left(\mathbf{x}^{\prime}, t\right)-\rho(\mathbf{x}, t)\right] /\right.$ $\partial t\} d V^{\prime}=0$ is canceled, given that $\partial G / \partial\left[\rho\left(\mathbf{x}^{\prime}, t\right)-\right.$ $\rho(\mathbf{x}, t)]=0$ for $\rho\left(\mathbf{x}^{\prime}, t\right) \neq \rho(\mathbf{x}, t)$, and $\partial\left[\rho\left(\mathbf{x}^{\prime}, t\right)-\right.$ $\rho(\mathbf{x}, t)] / \partial t=0$ for $\rho\left(\mathbf{x}^{\prime}, t\right)=\rho(\mathbf{x}, t)$. As a result, the time derivative of the right-hand side of $(22)$ is given by $(\partial / \partial t)$ $\int_{b(x, y)}^{z^{*}(\mathbf{x}, t)} A(z) d z=\left(\partial z^{*} / \partial t\right) A\left(z^{*}\right)+\int_{b(x, y)}^{z^{*}(\mathbf{x}, t)}[\partial A(z) / \partial t] d z=0$. Using $\partial A(z) / \partial t=0$, and noting that $A\left(z^{*}\right) \neq 0$ in the 
latter expression, we get $\partial z^{*} / \partial t=0$. Therefore, the second term in the integral on the right-hand side of (25) is omitted.

Substituting the density anomaly relation from (A4) into (25) yields

$$
\frac{\partial \mathrm{BPE}^{V}}{\partial t}=\int_{V} g z^{*} \frac{\partial \rho^{\prime}}{\partial t} d V=\int_{V} g z^{*}\left(\mathbf{\nabla} \mathbf{K} \nabla \rho^{\prime}-\mathbf{V} \cdot \nabla \rho^{\prime}\right) d V .
$$

Similar to previous studies (Hughes et al. 2009; Winters et al. 1995), we use $z^{*} \nabla \rho=\nabla \psi$, where $\psi=\int z^{*} d \rho$ to yield

$$
\begin{aligned}
\frac{\partial \mathrm{BPE}^{V}}{\partial t}= & -\int_{V} g\left[K_{h}\left|\nabla_{\mathbf{h}} \rho^{\prime}\right|^{2}+K_{z}\left(\frac{\partial \rho^{\prime}}{\partial z}\right)^{2}\right] \frac{\partial z^{*}}{\partial \rho^{\prime}} d V \\
& +\oint_{\Omega}\left[g z^{*}\left(K_{h} \nabla_{\mathbf{h}} \rho^{\prime}+K_{z} \frac{\partial \rho^{\prime}}{\partial z} \mathbf{z}\right)-g \psi \mathbf{V}\right] \cdot \mathbf{n}^{\Omega} d \Omega .
\end{aligned}
$$

As for the potential energy budget (13), the volume integral in (27) is the conversion rate of background potential energy owing to the irreversible diapycnal mixing (Winters et al. 1995; Hughes et al. 2009). At the reference state, the stratification is stable everywhere, and the derivative of $z^{*}$ with respect to $\rho^{\prime}$ is negative, so the contribution of irreversible mixing is a positive definite source of background potential energy. The terms in the surface integral in (27) are diffusive and advective fluxes of background potential energy across the envelope's surface of the domain. Using the boundary conditions (A7), (A13), and (A16), we get

$$
\begin{aligned}
\frac{\partial \mathrm{BPE}^{V}}{\partial t}= & \int_{\Omega^{H}}\left(\rho_{0} B_{0} z^{*}-g w \psi\right)^{H} d \Omega^{H} \\
& +\oint_{\Omega^{S}}\left(g z^{*} K_{h} \nabla_{h} \rho^{\prime}-g \psi \mathbf{V}\right)^{S} \cdot \mathbf{n}^{S} d \Omega^{S} \\
& -\int_{V} g\left[K_{h}\left|\nabla_{\mathbf{h}} \rho^{\prime}\right|^{2}+K_{z}\left(\frac{\partial \rho^{\prime}}{\partial z}\right)^{2}\right] \frac{\partial z^{*}}{\partial \rho} d V
\end{aligned}
$$

which is written in the abbreviated form as

$$
\frac{\partial \mathrm{BPE}^{V}}{\partial t}=\phi_{b B_{0}}^{H}+\phi_{b a}^{H}+\phi_{b d}^{S}+\phi_{b a}^{S}+\phi_{b d}^{V},
$$

where the $b$ subscript indicates contributors to the background potential energy budget. As evident from (22) and more clearly from (24), $z$ is dependent on the density field of the entire basin. Therefore, $z^{*}$ (and $\psi$ ) must be determined by an ordering/sorting code that is used for the calculations of (22) and (23). This makes the separation between the salinity and temperature contributions to the background potential energy, as it was done for the potential energy [i.e., (19)-(20)], impossible.
We are therefore limited to the calculation of the total background potential energy.

The available potential energy reservoir is given simply by the subtraction of the background potential energy from the potential energy:

$$
\mathrm{APE}^{V}=\mathrm{PE}^{V}-\mathrm{BPE}^{V}=\int_{V} \rho g\left(z-z^{*}\right) d V .
$$

Using (15) and (29), the evolution of the available potential energy is

$$
\frac{\partial \mathrm{APE}^{V}}{\partial t}=\phi_{a B_{0}}^{H}+\phi_{a a}^{H}+\phi_{a d}^{S}+\phi_{a a}^{S}-\phi_{p k}^{V}+\phi_{a d}^{V},
$$

where the $a$ subscript indicates contributors to the available potential energy budget.

\section{The energy budget of the Gulf of Eilat}

\section{a. Annual-mean view budget}

The set of (10), (15), (29), and (31) close the energy balance of the gulf, where (19) and (20) give the contributions of salinity and temperature variations to the potential energy budget. The different energetic pathways are illustrated schematically in Fig. 4, where their relative annual average contributions to the energy budget are indicated in the bracketed quantities (the percentages were calculated relative to the contribution of $\phi_{p B_{0}}^{H}$ ). In this figure, only terms important to the energy balance of the gulf are presented (such as $\phi_{p k}^{V}$ ), while small terms that contribute less than $\sim 0.1 \%$ (such as $\phi_{k \tau}^{B}$ ) are excluded, where the values of all terms are listed in Table 1. Similar to previous studies (Winters et al. 1995; Hughes et al. 2009), the irreversible mixing term $\left(\phi_{p d}^{V}\right)$ contributes directly to the background potential energy, and the transfer of energy from background to available potential energy is given via the term $\phi_{a d}^{V}$. Summations of the different contributors to each of the energetic reservoirs are nearly zero (Table 1), demonstrating that the model results represent a repeating seasonal cycle. One conclusion emerging from Fig. 4 is that on annual average the only positive contribution to $\mathrm{KE}^{V}$ comes from the reversible buoyancy flux term $\phi_{p k}^{V}$ (the conversion term from the available potential energy), while the main loss terms are the pressure work done at the strait $\phi_{k w}^{S}$, the shear dissipation $\left(\epsilon^{V}\right)$, and minor energy sink due to the wind stress at the surface $\phi_{k \tau}^{H}$. Negative values of $\phi_{k \tau}^{H}$ in the gulf are explained by the opposite surface flow and mean wind directions (section 1). As a result, the flow at the surface of the gulf does work against the wind stress forcing, and its contribution to the $\mathrm{KE}^{V}$ is negative on annual average. The fact 


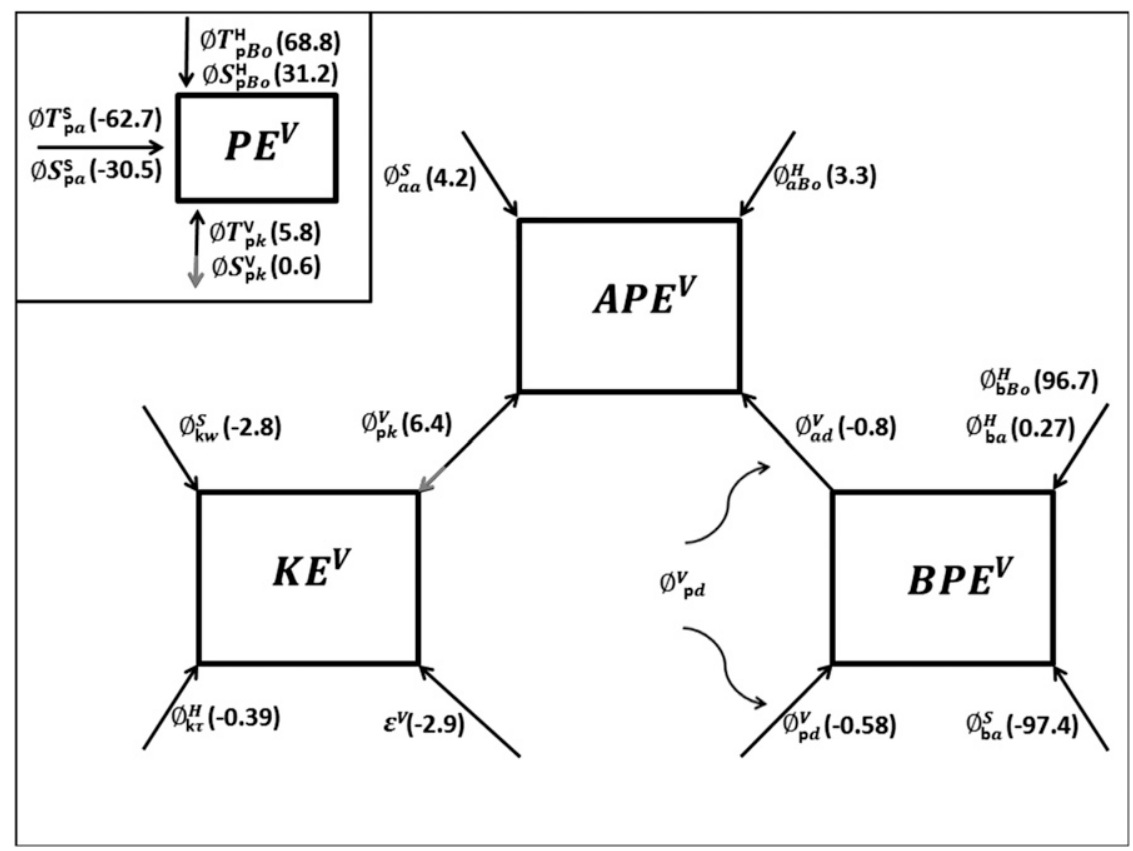

FIG. 4. Schematic representation of the dominant energetic pathways in the gulf expressed by (10), (15), (29), and (31). Energy is stored as $\mathrm{BPE}^{V}, \mathrm{APE}^{V}$, and $\mathrm{KE}^{V}$ reservoirs (the internal energy reservoir is not calculated in our model and therefore does not appear in this scheme). These energy reservoirs are influenced by 1) internal, volume-integrated, energy conversion terms $\left(\phi_{p k}^{V}, \epsilon^{V}, \phi_{p d}^{V}\right.$, and $\left.\phi_{a d}^{V}\right)$ and by 2) energy inputs at the basin's envelope that include the exchanges at the sea surface $\left(\phi_{a B_{0}}^{H}, \phi_{b B_{0}}^{H}, \phi_{b a}^{H}\right.$, and $\left.\phi_{k \tau}^{H}\right)$ and at the strait's cross section $\left(\phi_{a a}^{S}, \phi_{b a}^{S}\right.$, and $\left.\phi_{k w}^{S}\right)$. Bracketed quantities are the annual-mean values relative to $\phi_{p B_{0}}^{H}(\%)$. The arrows indicate the direction of the energy flow, when the assigned values are positive, with the exception of the term $\phi_{p k}^{V}$ that is indicated by the double arrow with the positive direction in gray. The inset at the upper-left corner shows the relative contributions of temperature and salinity fields to the potential energy as defined by (19)-(20). The values of all terms that appear in the balances of the different energy reservoir are listed in Table 1.

that the wind work subtracts energy to the buoyancydriven flow is similar to the Red Sea (e.g., Biton 2006), while in other semienclosed seas such as the Black Sea (Rachev and Stanev 1997) and the Mediterranean Sea (Korres et al. 2000), the wind work is a net and nonnegligible energy input to the circulation. As the only positive contribution to $\mathrm{KE}^{V}$ is coming from the energy transfers from the available potential energy (via the term $\phi_{p k}^{V}$ ), the first-order dynamics of the gulf (e.g., overturning circulation), and the submeso- to mesoscale dynamics, such as the chain of eddies that appear along its main axis, should all derive their energy from $\phi_{p k}^{V}$. On annual average, the energy supplied to the reversible buoyancy flux terms $\phi_{p k}^{V}$ is coming from the buoyancy flux at the surface $\phi_{a B_{o}}^{H}$ and the advection of available potential energy at the straits $\phi_{a a}^{S}$ (see the balance of $\mathrm{APE}^{V}$ in Fig. 4). Surprisingly, the largest source contribution to $\mathrm{APE}^{V}$ (and henceforth to $\mathrm{KE}^{V}$ ) is from $\phi_{a a}^{S}$, given that the dynamics of the gulf is largely induced by the conditions at the northern Red Sea. This result corresponds with the circulation of the gulf during the restratification period (April-August) proposed by Biton and Gildor (2011b), who showed that the entire circulation of the gulf during this period is driven by the hydrographic conditions at the northern Red Sea. The dominant terms in the budget of $\mathrm{BPE}^{V}$ are the contribution from the buoyancy flux at the surface $\phi_{b B_{0}}^{H}$ and the advection of background potential energy at the straits $\phi_{b a}^{S}$.

Last, we consider the relative contributions of temperature and salinity fields to the potential energy balance $\left[\partial \mathrm{PET}^{V} / \partial t\right.$ and $\partial \mathrm{PES}^{V} / \partial t$ calculated by (19)-(20)] as shown in Fig. 4. Similar to the case of the background potential energy, almost $100 \%$ of the energy source to the potential energy is due to the buoyancy flux from the surface of the gulf $\phi_{p B_{0}}^{H}$, where $\sim 70 \%$ of that energy is associated with the contribution of the heat flux to the buoyancy flux term $\phi T_{p B_{0}}^{H}$ and the rest is associated with the contribution of the net evaporation rate to buoyancy flux term $\phi S_{p B_{0}}^{H}$. Most of the energy sources from the buoyancy flux terms $\left(\phi_{p B_{0}}^{H}, \phi T_{p B_{0}}^{H}\right.$, and $\left.\phi S_{p B_{0}}^{H}\right)$ are 
TABLE 1. From left to right, bracketed quantities provide the relative annual average contributions to the energy budget of $\mathrm{PE}^{V}, \mathrm{PET}^{V}$, $\mathrm{PES}^{V}, \mathrm{BPE}^{V}, \mathrm{KE}^{V}$, and $\mathrm{APE}^{V}$ (in \%), respectively. Percentages were calculated with relative to the contribution of $\phi_{p B_{o}}^{H}\left(3.52 \times 10^{8} \mathrm{~J}\right.$ ). Values in the lower two rows indicate the sums of the total sources and sinks for each energy budget (in \%).

\begin{tabular}{|c|c|c|c|c|c|}
\hline $\mathrm{PE}^{V}$ & $\mathrm{PET}^{V}$ & $\mathrm{PES}^{V}$ & $\mathrm{BPE}^{V}$ & $\mathrm{KE}^{V}$ & $\mathrm{APE}^{V}$ \\
\hline$\phi_{p B_{0}}^{H}[100]$ & $\phi T_{p B_{0}}^{H}[68.8]$ & $\phi S_{p B_{0}}^{H}$ [31.2] & $\phi_{b B_{0}}^{H}[96.7]$ & $\phi_{p k}^{V}[6.4]$ & $-\phi_{p k}^{V}[-6.4]$ \\
\hline$\phi_{p a}^{S}[-93.2]$ & $\phi T_{p a}^{S}[-62.7]$ & $\phi S_{p a}^{S}[-30.5]$ & $\phi_{b a}^{S}[-97.4]$ & $\epsilon^{V}[-2.9]$ & $\phi_{a a}^{S}[4.2]$ \\
\hline$-\phi_{p k}^{V}[-6.4]$ & $-\phi T_{p k}^{V}[-5.8]$ & $-\phi S_{p k}^{V}[-0.6]$ & $\phi_{b a}^{H}[0.3]$ & $\phi_{k w}^{S}[-2.8]$ & $\phi_{a B_{0}}^{H}[3.3]$ \\
\hline$\phi_{p d}^{V}[-0.6]$ & $\phi T_{p d}^{V}[-0.7]$ & $\phi S_{p d}^{V}[0.1]$ & $\phi_{b d}^{V}[0.2]$ & $\phi_{k \tau}^{H}[-0.4]$ & $\phi_{a d}^{V}[-0.8]$ \\
\hline$\phi_{p a}^{H}[0.0]$ & $\phi T_{p a}^{H}[0.0]$ & $\phi S_{p a}^{H}[0.0]$ & $\phi_{b d}^{S}[0.0]$ & $\phi_{k a}^{H}[-0.2]$ & $\phi_{a a}^{H}[0.0]$ \\
\hline$\phi_{p d}^{S}[0.0]$ & $\phi T_{p d}^{S}[0.0]$ & $\phi S_{p d}^{S}[0.0]$ & - & $\phi_{k \tau}^{B}[-0.1]$ & $\phi_{a d}^{S}[0.0]$ \\
\hline- & - & - & - & $\phi_{k \tau}^{S}[0.0]$ & - \\
\hline - & - & - & - & $\phi_{k a}^{S}[0.0]$ & - \\
\hline - & - & - & - & $\phi_{k w}^{H}[0.0]$ & - \\
\hline 100 & 68.8 & 31.2 & 97.2 & 6.4 & 7.5 \\
\hline-100.2 & -69.1 & -31 & -97.4 & -6.5 & -7.2 \\
\hline
\end{tabular}

transferred to the advection of potential energy at the straits $(\sim 93.2 \%)$ and the rest by conversion to kinetic energy (via the term $\phi_{p k}^{V}$ ). Unexpectedly, there is a small potential energy loss of $-0.58 \%$ caused by the irreversible mixing term $\phi_{p d}^{V}$ that is composed by the loss of $-0.65 \%$ due to the term $\phi T_{p d}^{V}$ and a negligible energy gain of $0.07 \%$ due to the term $\phi S_{p d}^{V}$ (not shown). As we state in section 2, we used the nonlocal $K$-profile parameterization scheme for vertical mixing of Large et al. (1994). Therefore, the irreversible mixing term $\phi_{p d}^{V}$ accounts for implicit diffusion and the contributions of a variety of unresolved processes involved in vertical mixing, including convection. The negative values of $\phi_{p d}^{V}$ reflect mainly the loss of potential energy caused by thermally driven convection occurring during the mixing phase (September-March).

An important issue is the relative contributions of $\mathrm{PET}^{V}$ and $\mathrm{PES}^{V}$ to the dynamics of the gulf via the reversible buoyancy flux terms $\phi T_{p k}^{V}$ and $\phi S_{p k}^{V}$. As shown in Fig. 4, the net evaporation rate and heat flux from the surface of the gulf contribute at a ratio of 2:1 to the total buoyancy flux term at the surface. However, while the energy from evaporation is almost completely balanced by advection at the straits $\phi S_{p a}^{S}$, a larger fraction of the heat loss contribution transfers to the dynamic of the gulf, and the relative contribution to the dynamics via the terms $\phi T_{p k}^{V}$ and $\phi S_{p k}^{V}$ is about 10:1 (Fig. 4). Therefore, salinity is a minor contributor to the gulf dynamics, while major dynamic patterns observed in the gulf, such as the exchange flow with the northern Red Sea, the circulation driven by density differences, and stratification, are mainly caused by temperature changes, as noted in previous studies (Biton et al. 2008; Biton and Gildor 2011a,b,c).

\section{b. Seasonal view budget}

The seasonal energy balance in the gulf follows that of the annual mean (section 4a), yet it displays relations that are much more complicated. Unless stated otherwise in the following, the model results are based on 10day snapshots and cover conditions in the gulf over one seasonal cycle. The seasonal variations for all energy reservoirs are shown in Fig. 5. As expected for oceanic water (Pedlosky 1987; Gill 1982), most of the energy is stored in the background potential energy (largely following that of the total potential energy; Fig. 5a), while $\mathrm{KE}^{V}$ and $\mathrm{APE}^{V}$ values are six to seven orders of magnitude smaller than $\mathrm{PE}^{V}$. We next discuss the balance of the different energetic reservoirs.

\section{1) BALANCE OF THE POTENTIAL ENERGY AND BACKGROUND POTENTIAL ENERGY RESERVOIRS}

The dominant terms in the energy balances of $\mathrm{BPE}^{V}$, $\mathrm{PE}^{V}, \mathrm{PET}^{V}$, and $\mathrm{PES}^{V}$ are shown in Fig. 6 and include
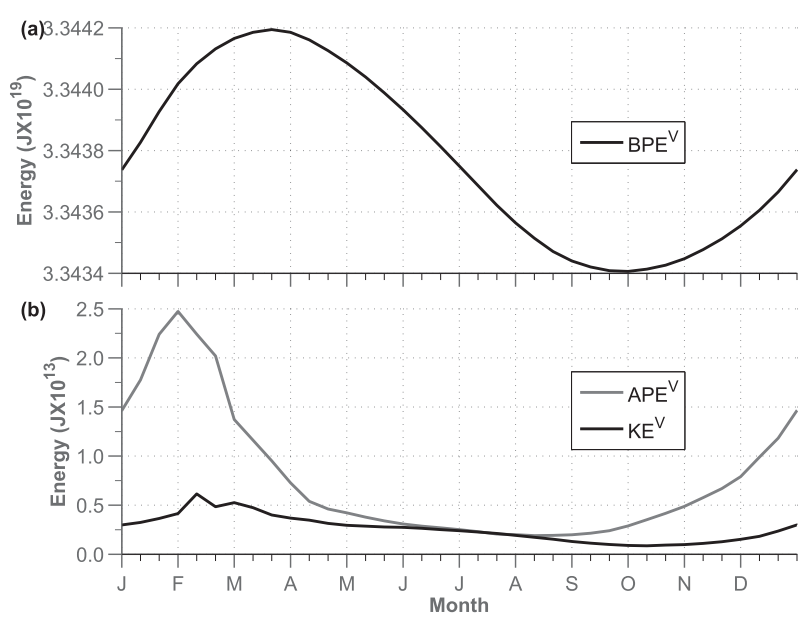

FIG. 5. Seasonal cycle (based on 10-day snapshot values) of (a) $\mathrm{BPE}^{V}$ (black) and of (b) $\mathrm{KE}^{V}$ (black) and $\mathrm{APE}^{V}$ (gray). Note that $\mathrm{PE}^{V}$ and $\mathrm{BPE}^{V}$ are indistinguishable; therefore, only $\mathrm{BPE}^{V}$ is presented in (a). 

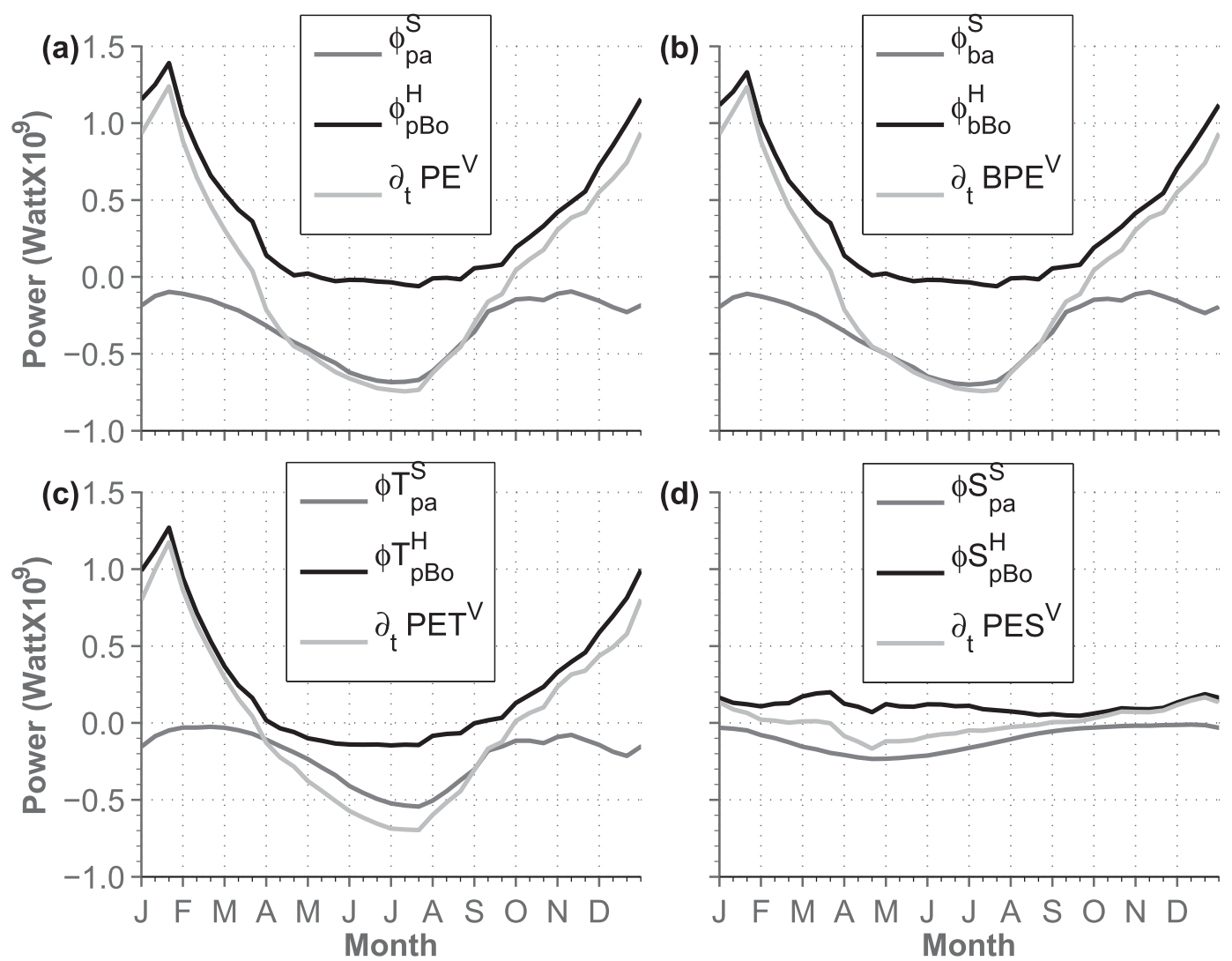

FIG. 6. Seasonal cycle (based on 10-day snapshot values) of the dominant terms in the balances of (a) $\mathrm{PE}^{V}$, (b) $\mathrm{BPE}^{V}$, (c) $\mathrm{PET}^{V}$, and (d) $\mathrm{PES}^{V}$. The lines in the different plots represent the seasonal advection of energy terms through the straits (dark gray), the buoyancy flux terms at the surface (black), and the balance of the energy reservoirs (light gray). The annual-mean values show that advection and buoyancy flux terms are nearly balanced on annual time scales (Fig. 4; Table 1); however, as shown here they are far from being in equilibrium on seasonal to monthly time scales.

the contributions of the buoyancy flux terms at the surface of the gulf (Fig. 6, solid black lines) compared with those from the advection terms of potential energy at the straits (Fig. 6, dark gray lines). The calculation of those terms is almost identical for $\mathrm{BPE}^{V}$ and $\mathrm{PE}^{V}$ and largely follows that of $\mathrm{PET}^{V}$. For this reason in the following discussion we mainly focus the contributions of temperature variations to the energy balance.

The advection terms of potential energy at the straits are a continuous sink of background potential energy and potential energy (Fig. 6, dark gray lines). This can be explained using the definition of the center of mass as follows:

$$
\begin{aligned}
\mathrm{PE}^{V} & =\int_{V} \rho g z d V=M g z_{\mathrm{cm}}, \quad \text { and } \\
\mathrm{BPE}^{V} & =\int_{V} \rho g z^{*} d V=M g z_{\mathrm{cm}}^{*},
\end{aligned}
$$

where $M$ is the total mass of the gulf, and $z_{\mathrm{cm}}$ and $z_{\mathrm{cm}}^{*}$ are the height of the center of mass of the gulf and the height of the center of mass of the gulf at the reference state, respectively. The influx of relatively less dense water from the northern Red Sea replaces the denser subsurface/intermediate water of the gulf. Therefore, the exchange flow at the straits tends to reduce $M$ and increase the stratification that lowered $z_{\mathrm{cm}}$, either way reducing the potential energy of the gulf. In contrast, buoyancy loss from the surface of the gulf increases the mean density of the gulf (and its total mass) and elevates $z_{\mathrm{cm}}$, either way increasing the potential energy of the gulf. Our model shows that the terms of the advection potential energy and background potential energy from the straits (i.e., $\phi_{p a}^{S}, \phi_{b a}^{S}, \phi T_{p a}^{S}$, and $\phi S_{p a}^{S}$ ) almost entirely balance the surface buoyancy flux terms on annual time scales (Fig. 4). However, the seasonality of the potential energy is generally dominated by only one of these terms at a time (Fig. 6a). The gulf gains potential energy during the mixing phase (September-March) from the buoyancy loss forcing and loses that energy through the advection term at the straits during the restratification phase 
(a)

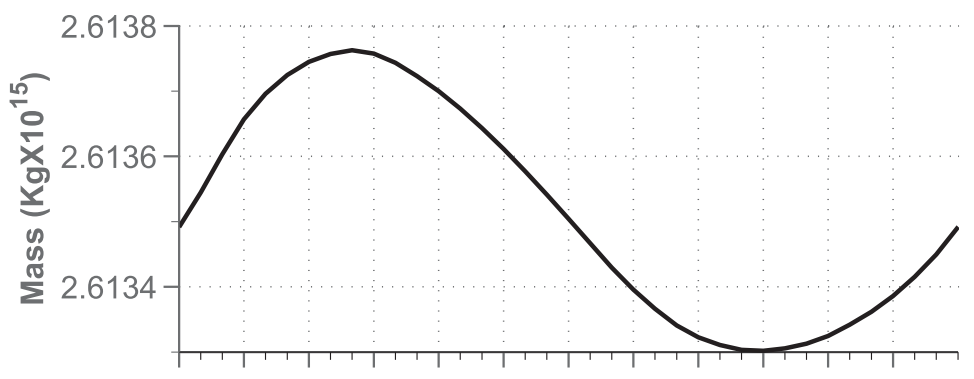

(b)

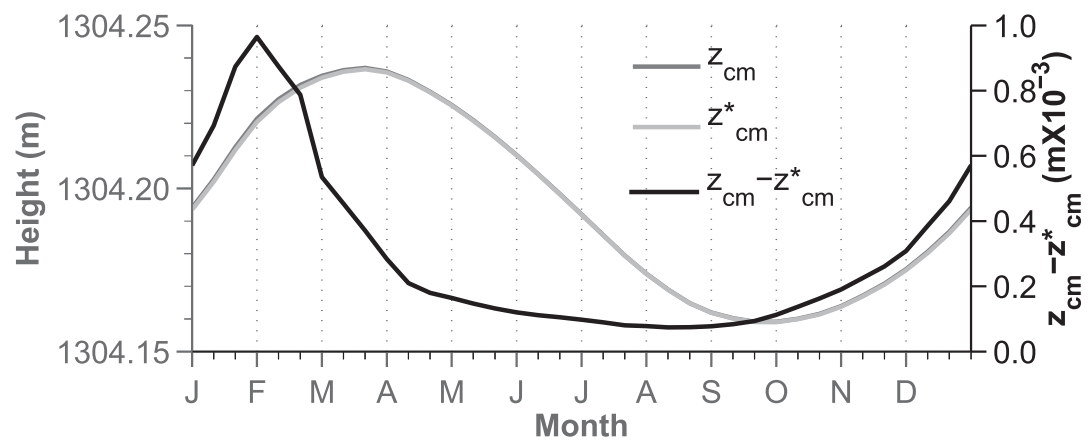

FIG. 7. Seasonal cycles (based on 10-day snapshot values) of (a) the total mass in the gulf $M$ (in $\mathrm{kg}$ ) caused by the seasonal cooling and warming of the surface layer of the gulf and (b) the height of the center of mass $z_{\mathrm{cm}}$ (dark gray line, left axis), height of the center of mass at the reference state $z_{\mathrm{cm}}^{*}$ (light gray line, left axis), and the different between the two heights $z_{\mathrm{cm}}-z_{\mathrm{cm}}^{*}$ (black line, right axis). The seasonality of $z_{\mathrm{cm}}-z_{\mathrm{cm}}^{*}$ reflects the variations in the baroclinic mode caused by the isopycnal tilting along gulf or by the formation of the along-gulf density gradient (section 4b), which dominate the seasonality of the available potential energy (see the comparison with the gray line in Fig. 5b). The baroclinic mode in the gulf develops due to baroclinic fluxes applied at the surface and through the straits and declines by irreversible mixing and a positive reversible buoyancy flux (section 4b). From September to February, $z_{\mathrm{cm}}-z_{\mathrm{cm}}^{*}$ is rebuilt due to the baroclinic flux at the surface (driven by the term $\phi_{a B_{0}}^{H}$ ). During February and March, the baroclinic flux at the surface is reduced, and it cannot sustain $z_{\mathrm{cm}}-z_{\mathrm{cm}}^{*}$ at peak values leading to the collapse and restoration of the system closer to its reference state mainly by adiabatic motions (section $4 b$ ).

(April-August), resulting in an almost perfect sinusoidal shape for the seasonality of the potential energy (Fig. 5a). It should be noted that $z_{\mathrm{cm}}, z_{\mathrm{cm}}^{*}, M, \mathrm{BPE}^{V}$, and $\mathrm{PE}^{V}$ display similar seasonality (Figs. 5, 7), yet the seasonality of $\mathrm{PE}^{V}$ and $\mathrm{BPE}^{V}$ are dominated more by $z_{\mathrm{cm}}$ variations rather than $M$ variations.

The potential energy balance as shown in Fig. 6a is analogous to the analysis of the heat budget of the gulf presented by Biton and Gildor (2011a,b,c) and can be explained similarly as follows: The advection of potential energy through the straits depends on the exchange flow rate (Fig. 8a) and on the density integrated over the straits' cross-sectional area [i.e., $\phi_{p a}^{S}=-\oint_{\Omega^{s}}\left(\rho^{\prime} g z \mathbf{V}\right)^{S} \cdot \mathbf{n}^{S} d \Omega^{S}$ as it appears in (14)-(15)]. While the density of the outflowing water from the gulf into the Red Sea does not change throughout the year, the northern Red Sea inflowing water warms monotonically from March to August (Fig. 8b, black), so the density differences between the exchange flow layers at the straits increase (Biton and Gildor 2011b). During this period a stabilizing atmospheric forcing exists (i.e., $\phi_{p B_{0}}^{H}<0$ ), and there is no force that can balance the advection of potential energy from the straits (Fig. 6). Therefore, the relatively dense gulf intermediate water exits the gulf (as during winter), but the surface layer of the gulf is refilled by the relatively warm and light northern Red Sea surface water influx. Because the gulf's area is small, the surface layer of the gulf is refilled on time scales of several months, and consequently the hydraulic conditions at the strait are changed, and the exchange flow rate is reduced during the restratification phase (Biton and Gildor 2011a). The net effect of the reduction of the exchange flow rate and the warming of the influx surface water during the restratification phase (Fig. 8) is a relatively large loss of advected potential energy through the straits during this period with maximal values in mid-July (Fig. 6a, dark gray line). Eventually, the whole upper $\sim 150 \mathrm{~m}$ in the entire gulf is replaced with warm northern Red Sea surface water during the restratification phase (Biton and Gildor 2011c), causing a decrease of the total mass and 
(a)

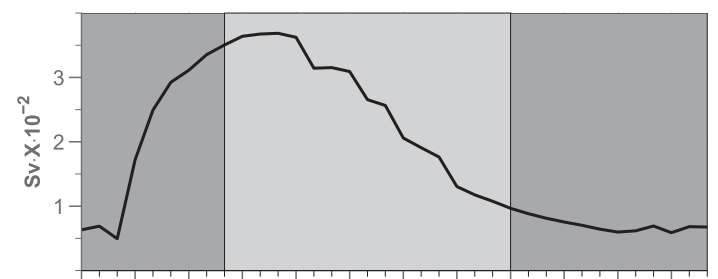

(b)

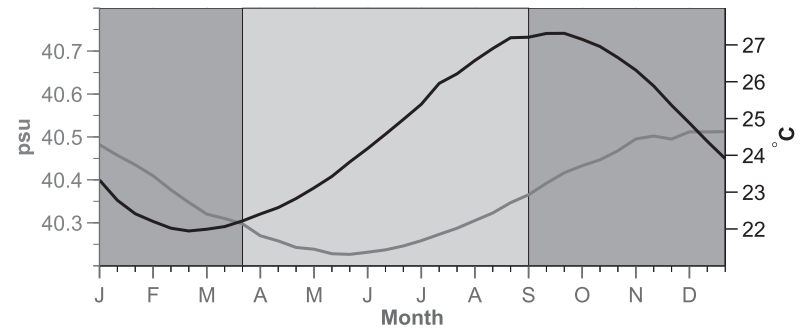

FIG. 8 . The 10-day average covering one simulated annual cycle of (a) the outflowing rate of the gulf intermediate water that reflects an approximate exchange flow in the straits (Biton and Gildor 2011b) and (b) temperature (black line, right axis) and salinity (gray line, left axis) of the inflowing northern Red Sea surface water. Mean salinity and temperature values for the outflowing gulf intermediate water are $\sim 40.7 \mathrm{psu}$ and $\sim 21.6^{\circ} \mathrm{C}$ year-round, respectively. Shaded areas indicate the seasonal mixing (dark gray) and restratification (light gray) phases in the evolution of the gulf stratification.

the height of center of mass (Fig. 7) and resulting in a potential energy reduction during this period as shown in Fig. 5a. It should be noted that the maximum values in the advection terms $\phi T_{p a}^{S}$ and $\phi S_{p a}^{S}$ appear at different times (Fig. 6) due to the phase difference between minimal salinity (May) and maximal temperature (September) in the northern Red Sea (Fig. 8b).

During the mixing phase the loss of potential energy due to the advection term is limited to small and nearly constant values, which can be explained by the integrated effects of the relatively weak exchange flow and cooling of the water entering the gulf from the northern Red Sea (Fig. 8). A small loss of energy due to the advected potential energy from September to November, despite the relatively high water temperature that enters the gulf, can be attributed to the minimum exchange flow rate during this time (Fig. 8a). From December to March the exchange flow increases, but the advected potential energy through the straits remains low because of the small temperature difference between the exchange flow layers. When the compensating loss of energy due to the advected potential energy from the straits is limited, the gulf gains potential energy by the buoyancy loss from the surface, and the potential energy of the gulf is rebuilt (Fig. 5). The buoyancy loss destabilizes the surface water and drives an efficient convective vertical mixing (mostly by the thermal mode) that takes place during the mixing phase (Fig. 2). Therefore, part of potential energy that is gained at the surface by $\phi_{p B_{0}}^{H}$ is lost by the convection process that is associated with the irreversible mixing term $\phi_{p d}^{V}$ (section $\left.4 \mathrm{a}\right)$.

\section{2) Budget of the AVAilable potential ENERGY RESERVOIR}

In section $4 a$, we show that on annual time scales the gulf dynamics are driven exclusively by the transfer available potential energy to kinetic energy (via $\phi_{p k}^{V}$ ). Therefore, to understand the dynamics we should first discuss the variations in the available potential energy. The seasonality of $\mathrm{APE}^{V}$ with respect to $\mathrm{KE}^{V}$ is shown in Fig. 5b, and the dominant terms that control their seasonality are shown in Figs. 9. $\mathrm{APE}^{V}$ shows much higher seasonal variability than $\mathrm{KE}^{V}$ (Fig. 5b), and a different seasonality with respect to $\mathrm{PE}^{V}$ and $\mathrm{BPE}^{V}$ (Fig. 5). Similar to $\mathrm{PE}^{V}, \mathrm{APE}^{V}$ increases between September and January, but it decreases over a short period between January and mid-March, just before the beginning of the restratification phase (Fig. 5b, gray line). Surprisingly, $\mathrm{KE}^{V}$ decreases slightly between January and mid-March, despite the large amount of energy transferred from $\mathrm{APE}^{V}$ during this period. As for $\mathrm{PE}^{V}$, we explain the available potential energy balance with the aid of the center of mass concept that is expressed by $\mathrm{APE}^{V}=M g\left(z_{\mathrm{cm}}-z_{\mathrm{cm}}^{*}\right)$. By definition, the background potential energy stores the minimum potential energy that is possible for a given density field, allowing only positive values for $z_{\mathrm{cm}}-z_{\mathrm{cm}}^{*}$. In addition, at the reference level all the isopycnals are leveled; therefore, the displacement between center of mass height of the gulf and its height at the reference level $\left(z_{\mathrm{cm}}-z_{\mathrm{cm}}^{*}\right)$ indicates the contribution of isopycnals vertical tilting in the gulf or the contributions of the baroclinic modes to the potential energy. Additionally, the isobars along the gulf are moderately vertically tilted along the gulf, when compared to isopycnals; therefore, $z_{\mathrm{cm}}-z_{\mathrm{cm}}^{*}$ is a measure of baroclinicity strength. The expression $z_{\mathrm{cm}}-z_{\mathrm{cm}}^{*}$ varies significantly throughout the year (reduced by $\sim 90 \%$ from its maximum to its minimum values; Fig. 7b); comparably, the total mass $M$ is only reduced by $0.01 \%$ from its maximum to its minimum values (Fig. 7a). Therefore, the seasonality of $\mathrm{APE}^{V}$ is expected to be dominated by the baroclinic variations in the gulf (i.e., $z_{\mathrm{cm}}-z_{\mathrm{cm}}^{*}$ ), as can be seen by the comparison of Fig. 5b (gray line) to Fig. 7b (black line). As such, the dominant terms that appear in the available potential energy balance in Fig. 9a affect mostly the energy stored in the baroclinic modes, but have little effect, if any, on the mass of the gulf (e.g., through net heating). We therefore consider the terms $\phi_{p k}^{V}, \phi_{a d}^{V}$, $\phi_{a B_{0}}^{H}$, and $\phi_{a a}^{S}$ as baroclinic energy flux terms to denote 

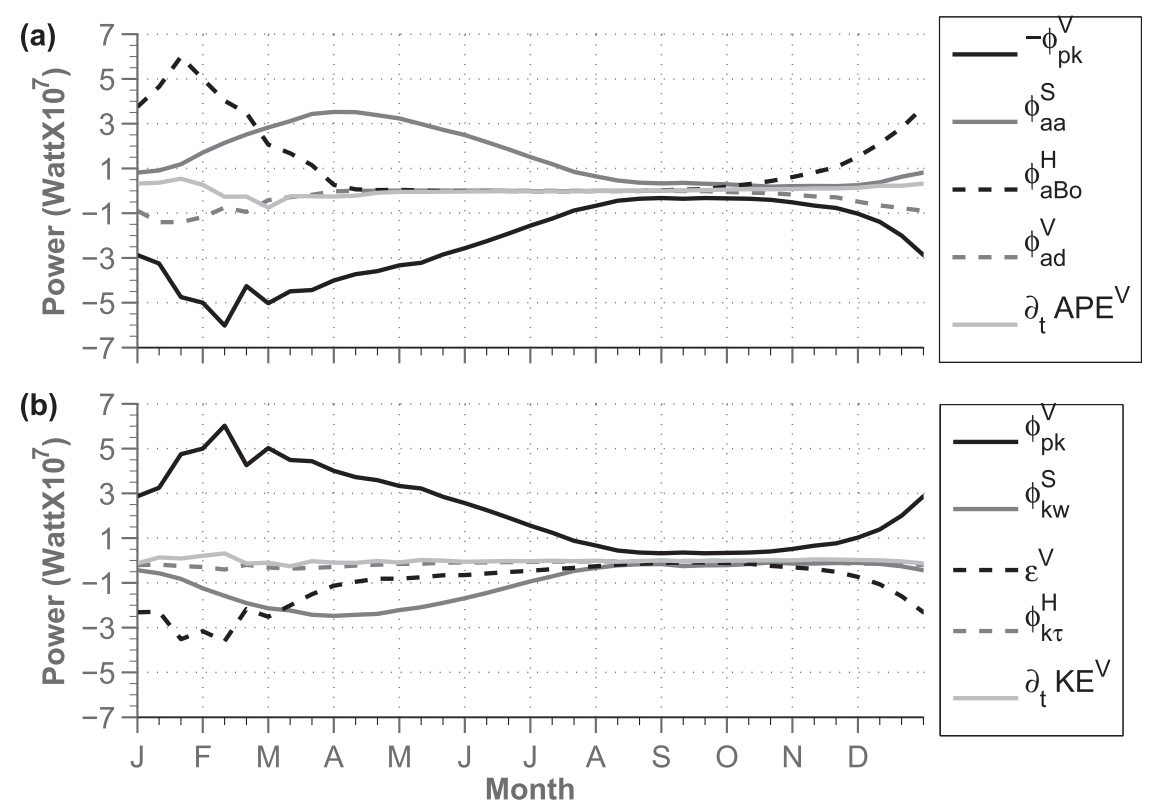

FIG. 9. Seasonal cycles of 10-day snapshots of the dominant terms contributions to the (a) $\mathrm{APE}^{V}$ and (b) $\mathrm{KE}^{V}$ reservoirs in the gulf that are defined in (31) and (10), respectively. The available potential energy budget in (a) is composed of the baroclinic fluxes associated with the reversible buoyancy flux (black line), advection through the straits (dark gray line), buoyancy flux at the sea surface (dashed black line), and irreversible mixing (dashed dark gray line). The kinetic energy budget in (b) is composed of the reversible buoyancy flux transferred from the available potential energy (black line), the pressure work done at the straits (dark gray line), the shear dissipation (dashed black line), and the wind stress at the surface (dashed dark gray line). The sum of the different components contributing to the budget of both energy reservoirs are indicated by light gray lines. As $\phi_{p k}^{V}$ is the only source for the kinetic energy, the horizontal circulation in the gulf is driven by vertical adiabatic motions that are forced by baroclinic fluxes at the straits and at the surface (the terms $\phi_{a a}^{S}$ and $\phi_{a B_{0}}^{H}$ ). Therefore, the horizontal circulation that includes the eddy formation along the gulf must be associated with deformation, divergence, and convergence processes. This relation is demonstrated by several idealized simulations as will be shown in a subsequent paper.

their contributions to the energy stored in the baroclinic modes of the gulf.

Probably the largest contribution to $z_{\mathrm{cm}}-z_{\mathrm{cm}}^{*}$, or to the baroclinic modes in the gulf, is due to the positive northward lateral density gradient (Fig. 2) and, to a lesser extent, to the eddy activity in the surface layer along the main axis of the gulf, while contributions from the subsurface layers to $z_{\mathrm{cm}}-z_{\mathrm{cm}}^{*}$ are negligible. The positive density gradient along the gulf in the surface layer is attributed to the less dense water of the northern Red Sea entering the gulf through the straits and by the buoyancy loss from the surface (only during the mixing phase) (Biton and Gildor 2011b). On the contrary, adiabatic subsidence of dense water from the surface layer and diapycnal mixing in the gulf tends to reduce the lateral density gradient or the isopycnal tilting and thus to reduce in baroclinicity strength. This view is in perfect analogy to the balance of the available potential energy as shown in Fig. 9a, where positive contributions to
$\mathrm{APE}^{V}$ are the advection term at the straits $\phi_{a a}^{S}$ and buoyancy flux at the surface $\phi_{a B_{0}}^{H}$, and negative contributors are the diapycnal irreversible mixing term $\phi_{a d}^{V}$ and the reversible buoyancy flux term $\phi_{p k}^{V}$.

Nonzero available potential energy needs to be supported by a continuous source of energy, otherwise, given enough time, dissipation terms [e.g., via baroclinic eddies as shown by Marshall et al. (2002)] will shift the system toward the reference state. Yet, locally changing the density field affects the entire reference state; therefore, shifting the system to the reference state needs the rearrangement of the density field in the entire basin. Therefore, $\mathrm{APE}^{V}$ is expected to be far from equilibrium with respect to its sources and sinks of energy, as shown in Fig. 5b (gray line). The minimum available potential energy at the end of the restratification phase is attributed to the weak density gradient and minimal tilting of the isopycnals along the gulf (Fig. 2). Between September and January, the dominant source for $\mathrm{APE}^{V}$ is the buoyancy 
flux term at the surface $\phi_{a B_{0}}^{H}$ (Fig. 9a, dashed black line). During this time the buoyancy loss from the surface builds up the density gradient along the gulf and the tilting of isopycnals along the gulf increases (Fig. 2), causing the observed increase in $\mathrm{APE}^{V}$ (Fig. 5b). Notably, the reversible buoyancy flux term and the irreversible mixing term respond immediately to the buoyancy flux term at the surface, with $\phi_{p k}^{V}$ as the dominant energy loss term. Positive values of $\phi_{p k}^{V}$ occur when the vertical adiabatic motion in the gulf mainly involves the sinking of dense water that is replaced by the upwelling of less dense water somewhere in the gulf. Such a process acts to level the isopycnals in the gulf or move the system closer to the reference state and therefore to a sink of available potential energy (Fig. 9a, black line). During a short period between January and mid-March, the surface layer in the gulf is entirely mixed (Fig. 2), the lateral density gradient collapses, and $\mathrm{APE}^{V}$ reduces from maximum to nearly minimum values (Fig. 5b, black line). Unexpectedly, the mixed conditions and the associated fast drop in $\mathrm{APE}^{V}$ occurring between January and mid-March are forced mainly by the sink term $\phi_{p k}^{V}$ and not by the irreversible mixing term $\phi_{a d}^{V}$ (Fig. 9a, black and dashed gray lines, respectively). As such, during this period the system is closely restored to its reference state mainly by adiabatic motions. This result is explained as follows: Since January, the source of available potential energy from the buoyancy flux at the surface drops quickly from its maximum values (Fig. 9, dashed black line). As a result, there is not enough incoming baroclinic energy flux to support the density gradient along the gulf, which therefore becomes unstable and collapses (adiabatically) to nearly reference state conditions.

During the restratification phase (mid-March to August), the integrated $\mathrm{APE}^{V}$ decreases moderately to its minimum seasonal value (Fig. 5b, gray line). Despite the relatively large variability in the total mass $M$, as for the mixing phase, $\mathrm{APE}^{V}$ is affected more by the reduction values of $z_{\mathrm{cm}}-z_{\mathrm{cm}}^{*}$, which is reduced by $\sim 50 \%$ during the restratification phase (Fig. 7b, black line). This time the balance of $\mathrm{APE}^{V}$ is composed of $\phi_{a a}^{S}$ and $-\phi_{p k}^{V}$ as the dominant source and sink terms, respectively (Fig. 9, dark gray and black lines). The loss of the energy by the term $-\phi_{p k}^{V}$ during the restratification phase can be partially explained by the adiabatic sinking of the dense intermediate water after the winter conditions (e.g., March conditions in Fig. 2), while it is replaced by the influx of warm water from the northern Red Sea. At the end of the mixing phase, the dense intermediate water accumulates up to the surface in the entire gulf (Fig. 2), while during the restratification phase it exits the gulf through the straits at much greater depths (170-250 m; Biton and Gildor 2011b) and therefore must sink while it moves southward to the straits. Overall, this replacement reduces the tilt of the isopycnals along the gulf causing a reduction in $\mathrm{APE}^{V}$. The explanation of the positive contribution by the term $\phi_{a a}^{S}$ to the available potential energy balance is more complicated. Unlike $\phi_{p a}^{S}$ that appears as a continuous sink in the balance of $\mathrm{PE}^{V}$ (Fig. 6a, dark gray line), $\phi_{a a}^{S}$ is a definite source of available potential energy. This somewhat unintuitive result can be explained by the result that the advection of potential energy reduces the potential energy in the gulf, yet it reduces the background potential energy by a greater amount of energy and therefore increases the available potential energy. The exchange flow at the straits affects the total mass and center of mass of the gulf $\left(M, z_{\mathrm{cm}}\right.$, and $\left.z_{\mathrm{cm}}^{*}\right)$ negatively, and therefore the positive contribution of $\phi_{a a}^{S}$ to $\mathrm{APE}^{V}$ must be associated with a positive contribution to $z_{\mathrm{cm}}-z_{\mathrm{cm}}^{*}$ or to the energy stored in the baroclinic modes in the gulf (i.e., increase of the along-gulf density gradient and isopycnal tilting). The water that enters the gulf has a lower density than the gulf's water, and therefore the exchange flow always advects as a density front or tilted isopycnal into the gulf so that it stores more energy in the baroclinic modes and subtracts energy from the reference state, which by definition is barotropic (where all density and pressure surfaces coincide; Pedlosky 1987). Being the only source of available potential energy during the restratification phase, the advection term $\phi_{a a}^{S}$ is also the only source of energy that drives the circulation in the gulf at this time. This result supports, quantitatively, the conclusion of Biton and Gildor (2011a,b,c) that the warming conditions in the northern Red Sea sustain the along-gulf density gradient and are responsible for the circulation in the gulf during the restratification phase.

\section{3) THE BUDGET OF THE KINETIC ENERGY RESERVOIR}

The sources and sinks of kinetic energy balance each other almost perfectly on seasonal time scales (Fig. 9b, black line), and therefore the kinetic energy reservoir shows relatively moderate seasonal variability (Fig. 5b, black line). Sinks of kinetic energy include dissipation $\epsilon^{V}$, pressure work at the straits $\phi_{k w}^{S}$, and wind stress at the surface $\phi_{k \tau}^{H}$, where the only source is $\phi_{p k}^{V}$ (Fig. 9b).

The immediate response of kinetic energy sink processes to the incoming energy from the reversible buoyancy flux term might suggest that these terms compensate each other locally. Yet, the connection between processes such as the pressure work that affects the balance at the straits and dissipation terms that include only the horizontal velocities (i.e., $\epsilon^{V}$ and $\phi_{k \tau}^{B}$ ) with the volumeintegrated vertical buoyancy flux term is nontrivial. Moreover, in our case the kinetic energy includes only the horizontal velocities [i.e., relation (1)], and therefore it is 
only stored in the horizontal circulation (1) that is composed of the mean northward flow, associated with the general circulation, and the chain of eddies along the gulf. The relations between the vertical adiabatic motion and horizontal circulation can be explained as follows: The terms $\phi_{a B_{0}}^{H}$ and $\phi_{a a}^{S}$ continuously maintain the energy stored in the baroclinic modes in the gulf (i.e., increase the available potential energy, isopycnal tilting, or horizontal density gradients), where part of that energy is transferred to vertical reversible adiabatic motion $\phi_{p k}^{V}$. This adiabatic motion is associated with deformation, divergence, and convergence processes that drive the horizontal circulation in the gulf. As the vertical adiabatic motion is the only source for the horizontal circulation, such relationships occur over the year, but the driving force for their creation is seasonally dependent. During the restratification phase, the entire source of energy that supports the adiabatic motion stems from $\phi_{a a}^{S}$ (Fig. 9a). By pushing warmer water into the gulf, $\phi_{a a}^{S}$ forces the front or the slanted isopycnals that separate the incoming and outgoing layers along the straits. As a result, the dense water of the gulf sinks in parallel to its exit through the straits, while it is replaced by the incoming warmer water from the northern Red Sea. In fact, $\phi_{a a}^{S}$ and $\phi_{k w}^{S}$ are similar throughout the year (Figs. 9a,b, dark gray lines), suggesting that the transfer of energy from $\phi_{a a}^{S}$ to $\phi_{p k}^{V}$ provides the entire energy necessary for the work done against the pressure gradient at the straits $\phi_{k w}^{S}$. During the mixing phase the main contribution to $\phi_{p k}^{V}$ is from $\phi_{a B_{0}}^{H}$ (Fig. 9a), where $\phi_{a B_{0}}^{H}$ slants the isopycnals and builds the density gradient along the gulf. This in turn drives vertical adiabatic motions that are immediately associated with the horizontal circulation. It should be emphasized that in $\phi_{a B_{0}}^{H}$ only the differential buoyancy flux is accounted for and not its effect on the total mass/average density of the gulf. More detailed findings on the relation between the energy transferred from the APE to KE via vertical adiabatic motions, the horizontal circulation that is characterized by the existence of successive pairs of cyclonic and anticyclonic eddies along the gulf, and the temperature/ density gradient in the upper layer of the gulf will be given in a subsequent paper.

\section{Conclusions}

We used oceanic model simulations and model-based energy budget calculations to qualitatively study the relative importance of different processes affecting the Gulf of Eilat dynamics and hydrographic conditions. The energy in the gulf changes mainly because of the seasonal mixing/cooling of the surface layer during SeptemberMarch that is followed by relatively fast warming when the dense and cold water of the gulf accumulated at the surface during winter is replaced by the water influx from the northern Red Sea. Most of the energy contributions at the surface and at the Straits of Tiran "radiate" to the reference state, which by its definition is barotropic, while much smaller fractions are stored in the available potential energy or in the baroclinic energy storage. The available potential energy is linked to the appearance of slanted isopycnals or baroclinicity that are associated with the along-gulf density/temperature gradient. This temperature gradient and its associated available potential energy reservoir is sustained by the baroclinic energy fluxes associated with the exchange at the straits that advects density fronts into the gulf (April-August) and by the differential heating at the surface (September-March), where part of that energy is converted to the dynamics (via $\phi_{p k}^{V}$ ) and to a lesser extent to background potential energy (via $\phi_{a d}^{V}$ ). During the year, the energy transferred from the available potential energy reservoir is the only source of kinetic energy, where losses of the kinetic energy are related to the pressure work done at the straits and energy dissipation. Therefore, it is the role of the available potential energy, or the energy stored in baroclinic modes, that controls the dynamics of the gulf, while contributions from other mechanical forcing, if any, are negligible. We found that temperature and salinity variations contribute to the dynamics via the energy transferred from the available potential energy by a ratio of 10:1. Therefore, salinity is a minor contributor to the gulf dynamics, while major dynamic patterns observed in the gulf are mainly affected by temperature changes as noted in previous studies (Biton et al. 2008; Biton and Gildor 2011b). Additionally, during the restratification phase the only contribution to the energy transferred to kinetic energy via the term $\phi_{p k}^{V}$, and thereby to the gulf's dynamics, comes from the baroclinic energy term at the straits $\phi_{a a}^{S}$, in agreement with the circulation of the gulf proposed by Biton and Gildor (2011a,b,c), which concluded that the entire circulation of the gulf during this period is driven by the hydrographic conditions in the northern Red Sea.

Acknowledgments. We thank two anonymous reviewers for useful comments. We are grateful to Miryam Freidman and Jack Silverman for technical help. HG is supported by the Israeli Ministry of Science and Technology. EB is partially supported by the North American Friends of IOLR (NAF-IOLR).

\section{APPENDIX A}

\section{Model Equations}

Under the conditions described in section 2 the model equations are as follows below. 
The horizontal momentum, vertical momentum, and continuity, written in Cartesian coordinates, are

$$
\frac{\partial \mathbf{V}_{h}}{\partial t}=-\mathbf{V} \cdot \boldsymbol{\nabla} \mathbf{V}_{h}-(2 \Omega \times \mathbf{V})_{h}-\frac{1}{\rho_{0}} \nabla_{h} P^{\prime}+\nabla \mathbf{A} \nabla \mathbf{V}_{h},
$$

$$
\frac{\partial P^{\prime}}{\partial z}=-g \rho^{\prime}, \quad \text { and }
$$

$$
\nabla \cdot \mathbf{V}=0 .
$$

In the above equations $\mathbf{V}=(u, v, w)$ is the velocity vector, $\mathbf{V}_{h}$ is its horizontal component, $\rho^{\prime}$ is the density anomaly with respect to the reference value of density $\rho_{0}, P^{\prime}$ is the pressure deviation from the pressure in a resting, hydrostatically balanced water column with constant density $\rho_{0}, g$ is the acceleration of gravity, and $\mathbf{A}=\left(A_{h}, A_{h}, A_{z}\right)$ is a diagonal tensor of eddy viscosity, where $A_{h}$ is the lateral eddy viscosity and $A_{z}$ is the vertical eddy viscosity. The eddy viscosity coefficients are calculated based from the resolved motions using the Smagorinsky parameterization (Smagorinsky 1963, 1993), with typical values ranging between 1 and $2 \mathrm{~m}^{2} \mathrm{~s}^{-1}$. We did not use any biharmonic viscosity terms, yet the model results are smooth and stable (e.g., current fields that are shown in Fig. 3).

The equations of temperature $T$, salinity $S$, and density anomaly $\rho^{\prime}$ are given by

$$
\frac{\partial\left(S, T, \rho^{\prime}\right)}{\partial t}=-\mathbf{V} \cdot \nabla\left(S, T, \rho^{\prime}\right)+\nabla \mathbf{K} \nabla\left(S, T, \rho^{\prime}\right),
$$

where $\mathbf{K}=\left(K_{h}, K_{h}, K_{z}\right)$ is a diagonal diffusion coefficient tensor, where $K_{h}$ and $K_{z}$ are the lateral and vertical diffusion coefficients, respectively. We apply $K_{h}=0$, and for the calculation of vertical diffusion, we use the nonlocal $K$-profile parameterization scheme for vertical mixing of Large et al. (1994), which unifies the treatments of a variety of unresolved processes involved in vertical mixing.

The equation of state is

$$
\begin{aligned}
\rho^{\prime} & =\rho(T, S, P)-\rho_{0} \\
& =\rho_{0}\left[-\alpha\left(T-T_{0}\right)+\beta\left(S-S_{0}\right)\right],
\end{aligned}
$$

where $\alpha$ is the thermal expansion coefficient, $\beta$ is the haline contraction coefficient, and $T_{0}$ and $S_{0}$ are the reference values for temperature and salinity, respectively. For the above equation we choose $\alpha=2.96 \times 10^{-4} \mathrm{~K}^{-1}$, $\beta=7.4 \times 10^{-4} \mathrm{psu}^{-1}, T_{0}=24^{\circ} \mathrm{C}, S_{0}=40.7 \mathrm{psu}$, and $\rho_{0}=$ $1028 \mathrm{~kg} \mathrm{~m}^{-3}$. For these values, the T-S diagram for the seawater properties in the gulf calculated using the linear equation of state (A5) and the nonlinear equation that was used in Biton and Gildor (2011b) is matched, ensuring that our model results agree with those of Biton and Gildor (2011b).

The positive direction along the vertical ( $z$ axis) is upward and changing between $b(x, y)<z<H+\eta(x, y)$, where $b(x, y)$ is the bottom elevation above a reference level, and $\eta(x, y)$ is the surface displacement from the elevation of water at rest $(z=H)$.

Integrating vertically, (A2) will give the relation of pressure anomaly $P^{\prime}$ :

$$
P^{\prime}=\rho_{o} g \eta+\int_{z}^{H} g \rho^{\prime} d z
$$

Note that in our case (the linear free-surface case), the right term is vertically integrated up to the surface reference level (i.e., $z=H$ ), short of reaching the actual surface height $\eta$.

In the above equations the horizontal flow ( $u$ and $v$ ), $T$, and $S$ are prognostic variables in the model, whereas diagnostic variables are the vertical flow $-w$ [calculated by integrating the continuity relation (A3) along the vertical axis], density anomaly using (A5), and pressure anomaly using (A6).

\section{Boundary conditions}

At the solid boundaries we apply no normal flow conditions:

$$
\mathbf{V} \cdot \mathbf{n}=0
$$

where $\mathbf{n}$ is a unit vector normal to the solid boundaries. For the tangential component $V_{T}$, we use a no-slip boundary condition for the bottom $\left(V_{T}=0\right)$ and slip conditions at the sides of the gulf $\left[\left(\partial V_{T} / \partial n\right)=0\right]$.

Since all gridcell faces of the model are normal compared to the velocity's components, the no normal flow conditions imply zero vertical velocity at the bottom $w[b(x, y)]=0$; therefore, the kinematic boundary conditions at the bottom reduce to

$$
\mathbf{V}_{h} \cdot \nabla_{h} b=0 .
$$

At the free surface, the kinematics boundary condition is

$$
\frac{\partial \eta}{\partial t}+\mathbf{V}_{h} \cdot \nabla_{h} \eta=w
$$

The equation for the free-surface $\eta$ derived by vertically integrating the continuity relation (A3) along with the kinematic boundary conditions (A8)-(A9) is

$$
\frac{\partial \eta}{\partial t}+\mathbf{V}_{h} \cdot \nabla_{h} \eta+\int_{b}^{\eta+H} \nabla_{h} \mathbf{V}_{h} d z=E
$$


Using Leibniz's theorem, we finally get

$$
\frac{\partial \eta}{\partial t}+\nabla_{h} \cdot \int_{b}^{H} \mathbf{V}_{h} d z=E
$$

where we incorporate a source term related to the net freshwater flux rate $E$ at the right-hand side of (A10), which acts at the surface. However, we are using virtual freshwater flux, where the net evaporation at the surface affects the salinity at the surface layer but not its volume balance; therefore, $E$ is replaced by 0 in the above equation.

Finally, the wind stress components $\tau=\left(\tau^{x}, \tau^{y}\right)$ are specified at the surface $(z=\eta+H \sim H)$,

$$
\frac{\partial(u, v)}{\partial z}=\frac{1}{\rho_{0} A_{z}}\left(\tau^{x}, \tau^{y}\right)
$$

and quadratic bottom drags are applied at the bottom $(z=b)$ :

$$
\frac{\partial(u, v)}{\partial z}=\frac{C_{d}\left|\mathbf{V}_{h}\right|}{\rho_{0} A_{z}}(u, v)
$$

where $C_{d}$ is the quadratic bottom drag coefficient (dimensionless). Note that the bottom drag represents an additional friction to that imposed by the no-slip condition at the bottom.

For the salinity, temperature, and density equations (A4) we use boundary conditions as follows below.

At the solid boundaries of the gulf we set the heat and salt fluxes to zero:

$$
K_{n} \frac{\partial(T, S)}{\partial n}=0
$$

At the surface $(z=H+\eta \sim H)$ we applied the net heat and freshwater fluxes ( $Q$ and $E$, respectively) in addition to small contributions of relaxation terms to sea surface salinity and temperature, as was specified in Biton and Gildor (2011b):

$$
\begin{gathered}
K_{z} \frac{\partial T}{\partial z}=-\Delta Z_{\text {surf }} \lambda_{T}\left(T-T_{0}\right)-\frac{Q}{\rho_{0} C_{w}} \equiv-\frac{Q^{*}}{\rho_{0} C_{w}}, \\
K_{z} \frac{\partial S}{\partial z}=-\Delta Z_{\text {surf }} \lambda_{S}\left(S-S_{0}\right)-S \cdot E \equiv-S \cdot E^{*},
\end{gathered}
$$

where $\Delta Z_{\text {surf }}$ is the thickness of the model's surface layer; $\lambda_{T}$ and $\lambda_{S}$ are the reciprocal relaxation time for temperature and salinity, respectively; $C_{w}$ is the heat capacity of seawater; and $Q^{*}$ and $E^{*}$ are the net heat and freshwater fluxes when the relaxation terms are included.
Last, using $K_{z}\left(\partial \rho^{\prime} / \partial z\right)=K_{z}\left\{\left[\left(\partial \rho^{\prime} / \partial T\right)(\partial T / \partial z)\right]+\right.$ $\left.\left[\left(\partial \rho^{\prime} / \partial S\right)(\partial S / \partial z)\right]\right\}$ and (A5), (A14), and (A15) yields the boundary conditions for the seawater density anomaly due to the thermohaline fluxes applied at the surface as follows:

$$
K_{z} \frac{\partial \rho^{\prime}}{\partial z}=\frac{B_{0} \rho_{0}}{g},
$$

where $B_{0}$ is the buoyancy flux at the surface that is given by

$$
B_{0}=\frac{g}{\rho_{0}}\left(\frac{\alpha Q^{*}}{C_{w}}+\rho_{0} \beta S E^{*}\right) .
$$

\section{APPENDIX B}

\section{Sensitivity to the Choice of Vertical Reference Level}

The calculated gravitational potential energy is sensitive to the choice of the vertical reference level. Yet, the dynamic should be invariant to that choice, as the conversions from the gravitational potential energy to other energy reservoirs in the system involve transformations from one gravitational potential energy state to another, and therefore the vertical reference level can be arbitrary chosen. This assures that all the potential energy terms involved in the dynamics, such as $\phi_{p k}^{V}$ and $\phi_{p d}^{V}$, and all terms that appear in the available potential energy equation should not be affected by the choices of the vertical reference level. To demonstrate this we rewrite (14) for an arbitrary vertical reference level:

$$
\begin{aligned}
\frac{\partial \mathrm{EP}^{V}}{\partial t}= & \left.\int_{\Omega^{\text {top }}} z_{\text {top }}\left(B_{0} \rho_{0}-\rho^{\prime} g w\right)\right|_{z=z_{\text {top }}} d \Omega^{\text {top }} \\
& +\oint_{\Omega^{S}}\left(g z K_{h} \nabla_{\mathbf{h}} \rho^{\prime}-\rho^{\prime} g z \mathbf{V}\right)^{S} \cdot \mathbf{n}^{S} d \Omega^{S} \\
& +\int_{V} g \rho^{\prime} w d V-\int_{V} g K_{v} \frac{\partial \rho^{\prime}}{\partial z} d V .
\end{aligned}
$$

As stated above, the terms $\phi_{p k}^{V}$ (the conversion term to kinetic energy) and $\phi_{p d}^{V}$ (contribute to the available potential energy) are left unchanged, yet $\phi_{p B_{0}}^{H}$ and $\phi_{p a}^{H}$ (the two terms in the left integral) are proportional to the vertical level of the surface of the ocean $z_{\text {top }}$, and therefore their values are sensitive to the choice of the vertical reference level. They equal zero only when the vertical reference level is chosen such that $z_{\text {top }}=0$ [equivalent to $z \in(\eta, b-H)]$. By its definition, the available potential energy is invariant to the choice of the vertical reference level as it depends on the difference $z-z^{*}$ (i.e., $\mathrm{APE}^{V}=\int_{V} \rho g\left(z-z^{*}\right) d V$ ) and as such only the 
background potential energy (the part that is not involved in the dynamics) is affected by the choice of the vertical reference level. Specifically, noting that $z^{*}$ depends only on the density field and that in general it is not equal to zero at the surface $\left(\left.z *\right|_{z_{\text {top }}} \neq 0\right)$, the term $\phi_{a B_{0}}^{\text {top }}=\int_{\Omega^{\text {top }}} B_{0} \rho_{0}\left(z_{\text {top }}-\left.z *\right|_{z_{\text {top }}}\right) d \Omega^{\text {top }}$ is invariant to the choice of the vertical reference level. In our energy budget calculations, we separated the gravitational potential energy into background and available potential energy. It can be seen from these calculations that in our case, when $z_{\text {top }}=H$ [equivalent to $z \in(\eta+H, b)$ ], most of the contribution of $\phi_{p B_{0}}^{H}$ is transferred to the background potential energy through the term $\phi_{b B_{0}}^{H}(96.7 \%$; Table 1$)$, while a much smaller portion is converted to available potential energy $\phi_{a B_{0}}^{H}(3.3 \%$; Table 1$)$. Equivalently, we can say that $100 \%$ of $\phi_{p B_{0}}^{H}$ had transferred to the background potential energy, where part of it $(3.3 \%)$ was converted to the available potential energy by the tilting of the isopycnal along the gulf due to the nonzero baroclinic term $\phi_{a B_{0}}^{H}$ (section $4 \mathrm{~b}$ ). In a similar way, in case that $z_{\text {top }}=0$ is chosen, $\phi_{a B_{0}}^{H}=3.3 \%, \phi_{b B_{0}}^{H}=-3.3 \%$, and $\phi_{p B_{0}}^{H}=0$.

\section{REFERENCES}

Ben-Sasson, M., M. S. Brenner, and N. Paldor, 2009: Estimating air-sea heat fluxes in semienclosed basins: The case of the Gulf of Elat (Aqaba). J. Phys. Oceanogr., 39, 185-202, doi:10.1175/ 2008JPO3858.1.

Berman, T., N. Paldor, and S. Brenner, 2000: Simulation of winddriven circulation in the Gulf of Elat (Aqaba). J. Mar. Syst., 26, 349-365, doi:10.1016/S0924-7963(00)00045-2.

,-- , and,- 2003 : The seasonality of tidal circulation in the Gulf of Elat. Isr. J. Earth Sci., 52, 11-19, doi:10.1560/ G72J-CYHM-D202-FJY2.

Biton, E., 2006: The Red Sea during the Last Glacial Maximum. M.S. thesis, Dept. of Environmental Science and Energy Research, Weizmann Institute of Science, 93 pp.

— through a strait and dynamics in a small convectively driven marginal sea: The Gulf of Aqaba (Gulf of Eilat). J. Geophys. Res., 116, C06017, doi:10.1029/2011JC006944.

$\longrightarrow$, and - 2011b: The general circulation of the Gulf of Aqaba (Gulf of Eilat) revisited: The interplay between the exchange flow through the Straits of Tiran and surface fluxes. J. Geophys. Res., 116, C08020, doi:10.1029/2010JC006860.

— evolution of salinity minimum in the Gulf of Aqaba (Gulf of Eilat). J. Geophys. Res., 116, C08022, doi:10.1029/2011JC007106.

_ J. Silverman, and H. Gildor, 2008: Observation and modeling of pulsating density current. Geophys. Res. Lett., 35, L14603, doi:10.1029/2008GL034123.

Cessi, P., N. Pinardi, and V. Lyubartsev, 2014: Energetics of semienclosed basins with two-layer flows at the straits. J. Phys. Oceanogr., 44, 967-979, doi:10.1175/JPO-D-13-0129.1.
Gill, A. E., 1982: Atmosphere-Ocean Dynamics. Academic Press, $662 \mathrm{pp}$.

Hall, J., and Z. Ben-Avraham, 1973: Bathymetric chart of the Gulf of Eilat. Ministry of Infrastructure, Geological Survey of Israel.

Huang, R. X., 1998: Mixing and available potential energy in a Boussinesq Ocean. J. Phys. Oceanogr., 28, 669-678, doi:10.1175/ 1520-0485(1998)028<0669:MAAPEI > 2.0.CO;2.

Hughes, G. O., A. M. Hogg, and R. W. Griffiths, 2009: Available potential energy and irreversible mixing in the meridional overturning circulation. J. Phys. Oceanogr., 39, 3130-3146, doi:10.1175/2009JPO4162.1.

Korres, G., N. Pinardi, and A. Lascaratos, 2000: The ocean response to low-frequency interannual atmospheric variability in the Mediterranean Sea. Part I: Sensitivity experiments and energy analysis. J. Climate, 13, 705-731, doi:10.1175/ 1520-0442(2000)013<0705:TORTLF $>2.0 . \mathrm{CO} ; 2$.

Kuhlbrodt, T., A. Griesel, M. Montoya, A. Levermann, M. Hofmann, and S. Rahmstorf, 2007: On the driving processes of the Atlantic meridional overturning circulation. Rev. Geophys., 45, RG2001, doi:10.1029/2004RG000166.

Large, W., J. McWilliams, and S. Doney, 1994: Oceanic vertical mixing: A review and a model with a nonlocal boundary layer parameterization. Rev. Geophys., 32, 363-403, doi:10.1029/ 94RG01872.

Manasrah, R. S., M. Badran, H. U. Lass, and W. Fennel, 2004: Circulation and winter deep-water formation in the northern Red Sea. Oceanologia, 46, 5-23.

Marshall, J., C. Hill, L. Perelman, and A. Adcroft, 1997a: A finitevolume, incompressible Navier Stokes model for studies of the ocean on parallel computers. J. Geophys. Res., 102, 5753-5766, doi:10.1029/96JC02775.

,,--- , and $\longrightarrow, 1997 \mathrm{~b}$ : Hydrostatic, quasi-hydrostatic, and nonhydrostatic ocean modeling. J. Geophys. Res., 102, 5733-5752, doi:10.1029/96JC02776.

— H. Jones, R. Karsten, and R. Wardle, 2002: Can eddies set ocean stratification? J. Phys. Oceanogr., 32, 26-38, doi:10.1175/ 1520-0485(2002)032<0026:CESOS>2.0.CO;2.

Pedlosky, J., 1987: Geophysical Fluid Dynamics. 2nd ed. SpringerVerlag, 710 pp.

Rachev, N. H., and V. Stanev, 1997: Eddy processes in semienclosed seas: A case study for the Black Sea. J. Phys. Oceanogr., 27, 1581-1601, doi:10.1175/1520-0485(1997)027<1581: EPISSA $>2.0 . \mathrm{CO} ; 2$.

Smagorinsky, J., 1963: General circulation experiments with the primitive equations. I. The basic experiment. Mon. Wea. Rev., 91, 99-164, doi:10.1175/1520-0493(1963)091<0099: GCEWTP $>2.3 . \mathrm{CO} ; 2$.

- 1993: Evolution of physical oceanography. Large Eddy Simulation of Complex Engineering and Geophysical Flows, B. Galperin and S. A. Orszag, Eds., Cambridge University Press, 3-36.

Tailleux, R., 2009: On the energetics of stratified turbulent mixing, irreversible thermodynamics, Boussinesq models, and the ocean heat engine controversy. J. Fluid Mech., 638, 339-382, doi:10.1017/S002211200999111X.

Winters, K. B., P. N. Lombard, J. J. Riley, and E. A. Dásaro, 1995: Available potential energy and mixing in densitystratified fluids. J. Fluid Mech., 289, 115-128, doi:10.1017/ S002211209500125X. 